Groups Geom. Dyn. 8 (2014), 391-414

DOI $10.4171 / \mathrm{GGD} / 231$
Groups, Geometry, and Dynamics

(C) European Mathematical Society

\title{
On hyperbolicity of free splitting and free factor complexes
}

\author{
Ilya Kapovich and Kasra Rafi*
}

\begin{abstract}
We show how to derive hyperbolicity of the free factor complex of $F_{N}$ from the Handel-Mosher proof of hyperbolicity of the free splitting complex of $F_{N}$, thus obtaining an alternative proof of a theorem of Bestvina-Feighn. We also show that under the natural map $\tau$ from the free splitting complex to free factor complex, a geodesic $[x, y]$ maps to a path that is uniformly Hausdorff-close to a geodesic $[\tau(x), \tau(y)]$.
\end{abstract}

Mathematics Subject Classification (2010). 20F65, 57M07.

Keywords. Free group, curve complex, outer automorphism group of the free group.

\section{Introduction}

The notion of a curve complex, introduced by Harvey [17] in late 1970s, plays a key role in the study of hyperbolic surfaces, mapping class group and the Teichmüller space.

If $S$ is a compact connected oriented surface, the curve complex $\mathscr{C}(S)$ of $S$ is a simplicial complex whose vertices are isotopy classes of essential non-peripheral simple closed curves. A collection $\left[\alpha_{0}\right], \ldots,\left[\alpha_{n}\right]$ of $(n+1)$ distinct vertices of $\mathcal{C}(S)$ spans an $n$-simplex in $\mathcal{C}(S)$ if there exist representatives $\alpha_{0}, \ldots, \alpha_{n}$ of these isotopy classes such that for all $i \neq j$ the curves $\alpha_{i}$ and $\alpha_{j}$ are disjoint. (The definition of $\mathcal{C}(S)$ is a little different for several surfaces of small genus). The complex $\mathcal{C}(S)$ is finite-dimensional but not locally finite, and it comes equipped with a natural action of the mapping class group $\operatorname{Mod}(S)$ by simplicial automorphisms. It turns out that the geometry of $\mathcal{C}(S)$ is closely related to the geometry of the Teichmüller space $\mathcal{T}(S)$ and also of the mapping class group itself. The curve complex is a basic tool in modern Teichmüller theory, and has also found numerous applications in the study of 3-manifolds and of Kleinian groups. A key general result of Masur and Minsky [23] says that the curve complex $\mathscr{C}(S)$, equipped with the simplicial metric, is a Gromov-hyperbolic space. Hyperbolicity of the curve complex was an important ingredient in the solution by Masur, Minsky, Brock and Canary of the

\footnotetext{
*The first author was partially supported by the NSF grant DMS-0904200. The second author was partially supported by the NSF grant DMS- 1007811 .
} 
Ending Lamination Conjecture [24], [27], [10] (see [26] for detailed background discussion about this solution).

The outer automorphism group $\operatorname{Out}\left(F_{N}\right)$ of a free group $F_{N}$ is a cousin of the mapping class group. However the group $\operatorname{Out}\left(F_{N}\right)$ is much less well understood and, in general, more difficult to study than the mapping class group. A free group analog of the Teichmüller space is the Culler-Vogtmann Outer space $\mathrm{cv}_{N}$, introduced by Culler and Vogtmann in [11]. The points of $\mathrm{cv}_{N}$ are free minimal discrete isometric actions of $F_{N}$ on $\mathbb{R}$-trees, considered up to $F_{N}$-equivariant isometry. The Outer space comes equipped with a natural action of $\operatorname{Out}\left(F_{N}\right)$. It is known that $\mathrm{cv}_{N}$ is finite-dimensional and contractible; as a result, quite a bit is known about homotopy properties of $\operatorname{Out}\left(F_{N}\right)$. However, the geometry of $\mathrm{cv}_{N}$ and of $\operatorname{Out}\left(F_{N}\right)$ proved to be much more difficult to tackle, particularly because $\mathrm{cv}_{N}$ lacks the various useful analytic and geometric structures present in the Teichmüller space case. Another problem is that many geometric dualities from the world of Riemann surfaces and their homeomorphisms break down for automorphisms of free groups.

In the case of a compact connected oriented surface $S$, an essential non-peripheral simple closed curve $\alpha$ on $S$ can be viewed in several other ways. Thus one can view $[\alpha]$ as a conjugacy class in the fundamental group $\pi_{1}(S)$. We may also think of $\alpha$ as corresponding to the (possibly disconnected) subsurface $K_{\alpha}$ of $S$ obtained by cutting $S$ open along $\alpha$. Third, $\alpha$ determines a splitting of $\pi_{1}(S)$ as an amalgamated product or an HNN-extension (depending on whether the curve $\alpha$ is separating or non-separating) over the infinite cyclic subgroup $\langle\alpha\rangle$. We can interpret adjacency of vertices in $\mathcal{C}(S)$ using each of these points of views - or a combination of them, providing several essentially equivalent descriptions of the curve complex. Thus two distinct vertices $[\alpha],[\beta]$ of $\mathcal{C}(S)$ are adjacent if and only if $\alpha$ is conjugate in $\pi_{1}(S)$ to an element of a vertex group of the cyclic splitting of $\pi_{1}(S)$ corresponding to $\beta$. Equivalently, $[\alpha]$ and $[\beta]$ of $\mathcal{C}(S)$ are adjacent if and only if the cyclic splittings of $\pi_{1}(S)$ corresponding to $[\alpha]$ and $[\beta]$ admit a common refinement, that is, a splitting of $\pi_{1}(S)$ as the fundamental group of a graph of groups with two edges and cyclic edge groups, such that collapsing one of the edges produces a splitting corresponding to $[\alpha]$ and collapsing the other edge produces a splitting corresponding to $[\beta]$. Also, $[\alpha]$ and $[\beta]$ are adjacent in $\mathcal{C}(S)$ if and only if there are connected components $K_{\alpha}^{\prime}$ of $K_{\alpha}$ and $K_{\beta}^{\prime}$ of $K_{\beta}$ such that $K_{\alpha}^{\prime} \subseteq K_{\beta}^{\prime}$ or $K_{\beta}^{\prime} \subseteq K_{\alpha}^{\prime}$.

In the case of $F_{N}$ these different points of view produce several possible analogs of the notion of a curve complex that are no longer essentially equivalent. The first of these is the free splitting complex $\mathrm{FS}_{N}$. The vertices of $\mathrm{FS}_{N}$ are nontrivial splittings of the type $F_{N}=\pi_{1}(\mathbb{A})$ where $\mathbb{A}$ is a graph of groups with a single edge (possibly a loop edge) and the trivial edge group; two such splittings are considered to be the same if their Bass-Serre covering trees are $F_{N}$-equivariantly isometric. Two distinct vertices $\mathbb{A}$ and $\mathbb{B}$ of $\mathrm{FS}_{N}$ are joined by an edge if these splittings admit a common refinement, that is, a splitting $F_{N}=\pi_{1}(\mathbb{D})$ where $\mathbb{D}$ is a graph of groups with two edges and trivial edge groups, such that collapsing one edge gives the splitting $\mathbb{A}$ and collapsing the other edge produces the splitting $\mathbb{B}$. Higher-dimensional simplices 
are defined in a similar way, see Definition 3.2 below for a careful formulation. For example, if $F_{N}=A * B * C$, where $A, B, C$ are nontrivial, then the splittings $F_{N}=(A * B) * C$ and $F_{N}=A *(B * C)$ are adjacent in $\mathrm{FS}_{N}$. There is a natural action of $\operatorname{Out}\left(F_{N}\right)$ on $\mathrm{FS}_{N}$ by simplicial automorphisms. The above definition of $\mathrm{FS}_{N}$ has a variation [29], called the edge-splitting complex, denoted $\mathrm{ES}_{N}$, where in the definition of vertices only splittings $\mathbb{A}$ with a single non-loop edge are allowed.

A rather different free group analog of the curve complex is the free factor complex $\mathrm{FF}_{N}$, originally introduced by Hatcher and Vogtmann [18]. The vertices of $\mathrm{FF}_{N}$ are conjugacy classes $[A]$ of proper free factors $A$ of $F_{N}$. Two distinct vertices $[A],[B]$ are joined by an edge in $\mathrm{FF}_{N}$ if there exist representatives $A$ of $[A]$ and $B$ of $[B]$ such that $A \leq B$ or $B \leq A$. Higher-dimensional simplices are defined similarly, see Definition 3.1 below. Note that this definition does not work well for $N=2$ as it produces a graph consisting of isolated vertices corresponding to conjugacy classes of primitive elements in $F_{2}$. However, there is a natural modification of the definition of $\mathrm{FF}_{N}$ for $N=2$ (see [4]) such that $\mathrm{FF}_{2}$ becomes the standard Farey graph (and in particular $\mathrm{FF}_{2}$ is hyperbolic).

A closely related object to $\mathrm{FF}_{N}$ is the simplicial intersection graph $I_{N}$. The graph $I_{N}$ is a bipartite graph with two types of vertices: single-edge free splittings $F_{N}=\pi_{1}(\mathbb{A})$ (that is, vertices of $\mathrm{FS}_{N}$ ) and conjugacy classes of simple elements of $F_{N}$. Here an element $a \in F_{N}$ is simple if $a$ belongs to some proper free factor of $F_{N}$. A free splitting $\mathbb{A}$ and a conjugacy class $[a]$ of a simple element $a$ are adjacent if $a$ is conjugate to an element of a vertex group of $\mathbb{A}$. The graph is a subgraph of a more general "intersection graph" defined in [20].

Both $\mathrm{FF}_{N}$ and $I_{N}$ admit natural $\operatorname{Out}\left(F_{N}\right)$-actions. It is also not hard to check that for $N \geq 3$ the graph $I_{N}$ is quasi-isometric to the free factor complex $\mathrm{FF}_{N}$. By contrast, the free factor complex $\mathrm{FF}_{N}$ and the free splitting complex $\mathrm{FS}_{N}$ are rather different objects geometrically. By construction, the vertex set $V\left(\mathrm{FS}_{N}\right)$ is a 1-dense subset of $V\left(I_{N}\right)$. Also, the inclusion map $\iota:\left(V\left(\mathrm{FS}_{N}\right), d_{\mathrm{FS}_{N}}\right) \rightarrow\left(I_{N}, d_{I_{N}}\right)$ is 2Lipschitz. However the distance between two free splittings in $I_{N}$ is generally much smaller than the distance between them in $\mathrm{FS}_{N}$. Intuitively, it is "much easier" for $\mathbb{A}$ and $\mathbb{B}$ to share a common elliptic simple element (which would make $d_{I_{N}}(\mathbb{A}, \mathbb{B}) \leq 2$ ) then for these splittings to admit a common refinement.

Until recently, basically nothing was known about the geometry of the above complexes. Several years ago Kapovich-Lustig [20] and Behrstock-Bestvina-Clay [2] showed that for $N \geq 3$ the (quasi-isometric) complexes $\mathrm{FF}_{N}$ and $I_{N}$ have infinite diameter. Since the inclusion map $\iota$ above is Lipschitz, this implies that $\mathrm{FS}_{N}$ has infinite diameter as well. A subsequent result of Bestvina-Feighn [3] implies that every fully irreducible element $\varphi \in \operatorname{Out}\left(F_{N}\right)$ acts on $\mathrm{FF}_{N}$ with positive asymptotic translation length (hence the same is true for the action of $\varphi$ on $\mathrm{FS}_{N}$ ). It is easy to see from the definitions that if $\varphi \in \operatorname{Out}\left(F_{N}\right)$ is not fully irreducible then some positive power of $\varphi$ fixes a vertex of $\mathrm{FF}_{N}$, so that $\varphi$ acts on $\mathrm{FF}_{N}$ with bounded orbits.

Sabalka and Savchuk proved [29] in 2010 that the edge-splitting complex $\mathrm{ES}_{N}$ is not Gromov-hyperbolic, because it possesses some quasi-flats. Aramayona and 
Souto [1] showed that every automorphism of $\mathrm{FS}_{N}$ is induced by some element of $\operatorname{Out}\left(F_{N}\right)$.

In 2011, two significant further advances occurred. First, Bestvina and Feighn [4] proved that for $N \geq 2$ the free splitting complex is Gromov-hyperbolic (as noted above, for $N=2$ this essentially follows from the definition of $\mathrm{FF}_{2}$, so the main case of the Bestvina-Feighn result is for $N \geq 3$ ). Then Handel and Mosher [16] proved that for all $N \geq 2$ the free splitting complex $\mathrm{FS}_{N}$ is also Gromov-hyperbolic. The two proofs are rather different in nature, although both are quite complicated. Recently Hilion and Horbez [19] produced another proof of hyperbolicity of $\mathrm{FS}_{N}$, using "surgery path" in the sphere complex model of $\mathrm{FS}_{N}$. Bestvina and Reynolds [6] and Hamenstädt [15] gave a description of the hyperbolic boundary of $\mathrm{FF}_{N}$. Also, Bestvina and Feighn [5] and then Sabalka and Savchuk [30] investigated analogs of subsurface projections in the $\mathrm{FS}_{N}$ and $\mathrm{FF}_{N}$ contexts.

In the present paper we show how to derive hyperbolicity of the free factor complex from the Handel-Mosher proof of hyperbolicity of the free splitting complex. This gives a new proof of the Bestvina-Feighn result [4] about hyperbolicity of $\mathrm{FF}_{N}$.

There is a natural "almost canonical" Lipschitz projection from the free splitting complex to a free factor complex. Namely, for any free splitting $v=\mathbb{A} \in V\left(\mathrm{FS}_{N}\right)$ choose a vertex $u$ of $\mathbb{A}$ and put $\tau(v):=\left[A_{u}\right]$, where $A_{u}$ is the vertex group of $u$ in $\mathbb{A}$. This defines a map (easily seen to be Lipschitz) $\tau: V\left(\mathrm{FS}_{N}\right) \rightarrow V\left(\mathrm{FF}_{N}\right)$. Extend this map to a graph-map $\tau: \mathrm{FS}_{N}^{(1)} \rightarrow \mathrm{FF}_{N}^{(1)}$ by sending every edge in $\mathrm{FS}_{N}$ to a geodesic in $\mathrm{FF}_{N}^{(1)}$ joining the $\tau$-images of the endpoints of that edge. Although the map $\tau$ is not quite canonically defined (since it involves choosing a vertex group in a free splitting $\mathbb{A}$ when defining $\tau(\mathbb{A}))$, it is easy to check that, for $N \geq 3$, if $\tau^{\prime}: V\left(\mathrm{FS}_{N}\right) \rightarrow V\left(\mathrm{FF}_{N}\right)$ is another map constructed by the above procedure, then $d\left(\tau(v), \tau^{\prime}(v)\right) \leq 2$ for all $v \in V\left(\mathrm{FS}_{N}\right)$.

We prove:

Theorem 1.1. Let $N \geq 3$. Then the free factor complex $\mathrm{FF}_{N}$ is Gromov-hyperbolic. Moreover, there exists a constant $C>0$ such that for any two vertices $x, y$ of $\mathrm{FS}_{N}$ and any geodesic $[x, y]$ in $\mathrm{FS}_{N}^{(1)}$ the path $\tau([x, y])$ is $C$-Hausdorff close to a geodesic $[\tau(x), \tau(y)]$ in $\mathrm{FF}_{N}^{(1)}$.

To prove Theorem 1.1, we first introduce a new object, called the free bases graph, and denoted $\mathrm{FB}_{N}$, see Definition 4.2 below. The vertices of $\mathrm{FB}_{N}$ are free bases of $F_{N}$, up to some natural equivalence. Informally, adjacency in $\mathrm{FB}_{N}$ corresponds to two free bases sharing a common element. We then prove (Proposition 4.3) that the natural map from $\mathrm{FB}_{N}$ to $\mathrm{FF}_{N}$ is a quasi-isometry. Thus to show that $\mathrm{FF}_{N}$ is hyperbolic it suffices to establish hyperbolicity of $\mathrm{FB}_{N}$. To do the latter we use a hyperbolicity criterion for graphs (Proposition 2.3 below) due to Bowditch [8]. Roughly, this criterion requires that there exists a family of paths $\mathscr{E}=\left\{g_{x, y}\right\}_{x, y}$ (where $x, y \in V X$ ) joining $x$ to $y$ and that there exists a "center"-like map $\Phi: V(X) \times V(X) \times V(X) \rightarrow V(X)$, such that 
the pair $(\mathscr{G}, \Phi)$ satisfies some nice "thin triangle" type properties, see Definition 2.2 below. Using Bowditch's criterion we obtain Corollary 2.4 saying that if $X, Y$ are connected graphs, with $X$ hyperbolic and if $f: X \rightarrow Y$ is a surjective Lipschitz graph-map with the property that if $d(f(x), f(y))$ is small then $f([x, y])$ has bounded diameter, then $Y$ is also hyperbolic. Moreover, in this case $f([x, y])$ is uniformly Hausdorff-close to any geodesic $[f(x), f(y)]$ in $Y$. (See also Theorem 3.11 in [25] and Proposition 3.1 in [9].)

We then construct a surjective Lipschitz map $f: \mathrm{FS}_{N}^{\prime} \rightarrow \mathrm{FB}_{N}$, where $\mathrm{FS}_{N}^{\prime}$ is the barycentric subdivision of $\mathrm{FS}_{N}$. The map $f$ restricts to a natural bijection from a subset $S$ of $V\left(\mathrm{FS}_{N}^{\prime}\right)$, corresponding to $N$-roses, to the set $V\left(\mathrm{FB}_{N}\right)$ of vertices of $\mathrm{FB}_{N}$. Thus we may, by abuse of notation, say that $S=V\left(\mathrm{FB}_{N}\right)$ and that $\left.f\right|_{S}=\operatorname{Id}_{S}$. In [16] Handel and Mosher constructed nice paths $g_{x, y}$ given by "folding sequences" between arbitrary vertices $x$ and $y$ of $\mathrm{FS}_{N}^{\prime}$, and proved that these paths are quasigeodesics in $\mathrm{FS}_{N}^{\prime}$. To apply Corollary 2.4 to the map $f: \mathrm{FS}_{N}^{\prime} \rightarrow \mathrm{FB}_{N}$ it turns out to be enough to show that $f\left(g_{x, y}\right)$ has bounded diameter if $x, y \in S$ and $d(f(x), f(y)) \leq 1$ in $\mathrm{FB}_{N}$. To do that we analyze the properties of the Handel-Mosher folding sequences in this specific situation. The construction of $g_{x, y}$ for arbitrary $x, y \in V\left(\mathrm{FS}_{N}^{\prime}\right)$ is fairly complicated. However, in our situation, we have $x, y \in S$, so that $x, y$ correspond to free bases of $F_{N}$. In this case the construction of $g_{x, y}$ becomes much easier and boils down to using standard Stallings foldings (in the sense of [21], [31]) to get from $x$ to $y$. Verifying that $f\left(g_{x, y}\right)$ has bounded diameter in $\mathrm{FB}_{N}$, assuming $d(f(x), f(y)) \leq 1$, becomes a much simpler task. Thus we are able to conclude that $\mathrm{FB}_{N}$ is Gromov-hyperbolic, and, moreover, that $f([x, y])$ is uniformly Hausdorffclose to any geodesic $[f(x), f(y)]$ in $\mathrm{FB}_{N}$. Using the quasi-isometry between $\mathrm{FB}_{N}$ and $\mathrm{FF}_{N}$ provided by Proposition 4.3, we then obtain the conclusion of Theorem 1.1.

Moreover, as we note in Remark 6.2, our proof of Theorem 1.1 provides a fairly explicit description of quasigeodesics joining arbitrary vertices (i.e. free bases) in $\mathrm{FB}_{N}$ in terms of Stallings foldings.

Acknowledgement. This paper grew out of our discussions during the November 2011 AIM SQuaRE Workshop "Subgroups of $\operatorname{Out}\left(F_{N}\right)$ and their action on outer space" at the American Institute of Mathematics in Palo Alto. We thank AIM and the other participants of the workshop, Thierry Coulbois, Matt Clay, Arnaud Hilion, Martin Lustig and Alexandra Pettet, for the stimulating research environment at the workshop. We are also grateful to Patrick Reynolds for the many helpful comments on the initial version of this paper and to Saul Schleimer for a helpful discussion regarding references for Proposition 2.6. We especially thank the referee for the numerous useful comments and suggestions, and for providing Figure 1 (see Section 6 below) illustrating the relationships and the maps between the main objects considered in this paper. 


\section{Hyperbolicity criteria for graphs}

Convention 2.1. From now on, unless specified otherwise, every connected graph $X$ will be considered as a geodesic metric space with the simplicial metric (where every edge has length 1). As in the introduction, we denote the vertex set of $X$ by $V(X)$. Also, when talking about a connected simplicial complex $Z$ as a metric space, we will in fact mean the 1 -skeleton $Z^{(1)}$ of $Z$ endowed with the simplicial metric.

Let $X, Y$ be connected graphs. A graph-map from $X$ to $Y$ is a continuous function $f: X \rightarrow Y$ such that $f(V(X)) \subseteq V(Y)$ (so that $f$ takes vertices to vertices), and such that for every edge $e$ of $X f(e)$ is an edge-path in $Y$ (where we allow for an edge-path to be degenerate and to consist of a single vertex). Note that if $f: X \rightarrow Y$ is a graph-map and $X^{\prime}$ is a subgraph of $X$ then $\left.f\right|_{X^{\prime}}: X^{\prime} \rightarrow Y$ is also a graph-map and $f\left(X^{\prime}\right)$ is a subgraph of $Y$.

We say that a graph-map $f: X \rightarrow Y$ is L-Lipschitz (where $L \geq 0$ ) if for every edge $e$ of $X$ the edge-path $f(e)$ has simplicial length $\leq L$.

We use a characterization of hyperbolicity for a geodesic metric space $\left(X, d_{X}\right)$ that is due to Bowditch [8]. A similar hyperbolicity conditions have been originally stated by Masur and Minsky (see Theorem 2.3 in [23]). A related statement was also obtained by Hamenstädt [14].

Definition 2.2 (Thin triangles structure). Let $X$ be a connected graph. Let $\mathscr{E}=$ $\left\{g_{x, y} \mid x, y \in V(X)\right\}$ be a family of edge-paths in $X$ such that for any vertices $x, y$ of $X g_{x, y}$ is a path from $x$ to $y$ in $X$. Let $\Phi: V(X) \times V(X) \times V(X) \rightarrow V(X)$ be a function such that for any $a, b, c \in V(X)$,

$$
\Phi(a, b, c)=\Phi(b, c, a)=\Phi(c, a, b) .
$$

Assume, for constants $B_{1}$ and $B_{2}$ that $\mathcal{E}$ and $\Phi$ have the following properties:

(1) For $x, y \in V(X)$, the Hausdorff distance between $g_{x, y}$ and $g_{y, x}$ is at most $B_{2}$.

(2) For $x, y \in V(X), g_{x, y}:[0, l] \rightarrow X, s, t \in[0, l]$ and $a, b \in V(X)$, assume that

$$
d_{X}(a, g(s)) \leq B_{1} \quad \text { and } \quad d_{X}(b, g(t)) \leq B_{1} .
$$

Then, the Hausdorff distance between $g_{a, b}$ and $\left.g_{x, y}\right|_{[s, t]}$ is at most $B_{2}$.

(3) For any $a, b, c \in V(X)$, the vertex $\Phi(a, b, c)$ is contained in a $B_{2}$-neighborhood of $g_{a, b}$.

Then, we say that the pair $(\mathscr{E}, \Phi)$ is a $\left(B_{1}, B_{2}\right)$-thin triangles structure on $X$.

The following result is a slightly restated special case of Proposition 3.1 in [8].

Proposition 2.3 (Bowditch). Let $X$ be a connected graph. For every $B_{1}>0$ and $B_{2}>0$, there is $\delta>0$ and $H>0$ so that if $(\mathcal{E}, \Phi)$ is a $\left(B_{1}, B_{2}\right)$-thin triangles structure on $X$ then $X$ is $\delta$-hyperbolic. Moreover, every path $g_{x, y}$ in $\mathcal{E}$ is $H$-Hausdorf-close to any geodesic segment $[x, y]$. 
Corollary 2.4. Let $X$ and $Y$ be connected graphs and assume that $X$ is $\delta_{0}$-Gromovhyperbolic. Let $f: X \rightarrow Y$ be a L-Lipschitz graph-map and $f(V(X))=V(Y)$. Suppose there are integers $M_{1}>0$ and $M_{2}>0$ so that, for $x, y \in V(X)$, if $d_{Y}(f(x), f(y)) \leq M_{1}$ then $\operatorname{diam}_{Y} f([x, y]) \leq M_{2}$.

Then, there exists $\delta_{1}>0$ such that $Y$ is $\delta_{1}$-hyperbolic. Moreover, there exists $H>0$ such that for any vertices $x, y$ of $X$ the path $f([x, y])$ is H-Hausdorff close to any geodesic $[f(x), f(y)]$ in $Y$.

Proof. For every pair of vertices $a, b \in X$, let $g_{a, b}$ be any geodesic segment $[a, b]$ and let $\mathcal{G}$ be the set of all these paths. Also, for any vertices $a, b, c$ of $X$ let

$$
\Phi(a, b, c)=\Phi(b, c, a)=\Phi(c, a, b)
$$

be any vertex of $X$ that is at most $\delta_{0}$ away from each of $[a, b],[b, c],[a, c]$. The hyperbolicity of $X$ implies that $(\mathcal{E}, \Phi)$ forms a $\left(b_{1}, b_{2}\right)$-thin triangles structure on $X$ for some $b_{1}$ and $b_{2}$ depending on $\delta_{0}$. We now push this structure $(\mathcal{E}, \Phi)$ forward via the map $f$.

For any vertex $y$ of $Y$ choose a vertex $v_{y}$ of $X$ such that $f\left(v_{y}\right)=y$.

For any vertices $y, z \in Y$ choose a geodesic $g_{v_{y}, v_{z}}$ from $v_{y}$ to $v_{z}$ in $X$ (note that such a geodesic is generally not unique) and let $g_{y, z}^{\prime}:=f\left(g_{v_{y}, v_{z}}\right)$. Then let $\mathcal{E}^{\prime}=\left\{g_{y, z}^{\prime} \mid y, z \in V(Y)\right\}$. Now, for any vertices $w, y, z \in Y$ put

$$
\Phi^{\prime}(w, y, z):=f\left(\Phi\left(v_{w}, v_{y}, v_{z}\right)\right) .
$$

We claim that, the pair $\left(\mathcal{E}^{\prime}, \Phi^{\prime}\right)$ is a $\left(B_{1}, B_{2}\right)$-thin triangles structure for $Y$ for some $B_{1}$ and $B_{2}$. The conditions (1) and (3) of Definition 2.2 are satisfied as long as $B_{2} \geq L b_{2}$ since $f$ is $L$-Lipschitz. Thus we only need to verify that condition (2) of Definition 2.2 holds for $\left(\mathscr{G}^{\prime}, \Phi^{\prime}\right)$.

Let $y, z$ be vertices of $Y, v_{y}, v_{z}$ be the associated vertices in $X, g_{v_{y}, v_{z}}:[0, l] \rightarrow X$ be the path in $\mathcal{G}_{\text {connecting }} v_{x}$ to $v_{y}$ and $g_{y, z}^{\prime}:\left[0, l^{\prime}\right] \rightarrow Y$ be the $f$-image of $g_{v_{y}, v_{z}}$. In the interest of brevity, we denote these paths simply by $g$ and $g^{\prime}$.

Let $B_{1}=M_{1}$ and let, $a, b \in Y$ and $s^{\prime}, t^{\prime} \in\left[0, l^{\prime}\right]$ be such that

$$
d_{Y}\left(a, g^{\prime}\left(s^{\prime}\right)\right) \leq B_{1} \quad \text { and } \quad d_{Y}\left(b, g^{\prime}\left(t^{\prime}\right)\right) \leq B_{1} .
$$

We need to bound the Hausdorff distance between $g_{a, b}^{\prime}=f\left(g_{v_{a}, v_{b}}\right)$ and $\left.g^{\prime}\right|_{\left[s^{\prime}, t^{\prime}\right]}$.

Let $s, t \in[0, l]$ be such that $f g(s)=g^{\prime}\left(s^{\prime}\right)$ and $f g(t)=g^{\prime}\left(t^{\prime}\right)$. Let $u$ be a vertex of $g_{a, b}^{\prime}$. From hyperbolicity, we have $v_{u}$ is contained in a $2 \delta_{0}$-neighborhood of the union

$$
\left.g\right|_{[s, t]} \cup\left[g(s), v_{a}\right] \cup\left[g(t), v_{b}\right] .
$$

Thus $u$ is $\left(2 L \delta_{0}\right)$-close to the union

$$
\left.g^{\prime}\right|_{\left[s^{\prime}, t^{\prime}\right]} \cup f\left(\left[g(s), v_{a}\right]\right) \cup f\left(\left[g(t), v_{b}\right]\right) .
$$


But the $d_{Y}$-diameters of $f\left(\left[g(s), v_{a}\right]\right) f\left(\left[g(t), v_{b}\right]\right)$ are less than $M_{2}$. Hence, $u$ is in $\left(2 L \delta_{0}+M_{2}\right)$-neighborhood of $\left.g^{\prime}\right|_{\left[s^{\prime}, t^{\prime}\right]}$. Similarly, $\left.g^{\prime}\right|_{\left[s^{\prime}, t^{\prime}\right]}$ is in the same size neighborhood of $g_{a, b}^{\prime}$. The condition (2) of Definition 2.2 holds for $B_{2}=$ $\left(2 L \delta_{0}+M_{2}\right)$.

Therefore, by Proposition 2.3, the graph $Y$ is $\delta_{1}$-hyperbolic, and, moreover, for any two vertices $y, z$ of $Y$ the path $g_{y, z}^{\prime}=f\left(g_{v_{y}, v_{z}}\right)$ is $H^{\prime}$-Hausdorff close to $[y, z]$ for some constant $H^{\prime} \geq 0$ independent of $y, z$. Since $g_{v_{y}, v_{z}}$ was chosen to be a geodesic from $v_{y}$ to $v_{z}$ in $X$ and any two such geodesics are $\delta_{0}$-Hausdorff close, by increasing the constant $H^{\prime}$ we also get that for any geodesic $[y, z]$ from $y$ to $z$ in $Y$ and any geodesic $\left[v_{y}, v_{z}\right]$ from $v_{y}$ to $v_{z}$ in $X$ the paths $[y, z]$ and $f\left(\left[v_{y}, v_{z}\right]\right)$ are $H^{\prime}$-Hausdorff close in $Y$.

Now let $y, z$ be any vertices of $Y$ and let $v_{y}^{\prime}, v_{z}^{\prime}$ be arbitrary vertices of $X$ such that $f\left(v_{y}^{\prime}\right)=y$ and $f\left(v_{z}^{\prime}\right)=z$. A geodesic $\left[v_{y}^{\prime}, v_{z}^{\prime}\right]$ in $X$ is contained in the $2 \delta_{0^{-}}$ neighborhood of $\left[v_{y}^{\prime}, v_{y}\right] \cup\left[v_{y}, v_{z}\right] \cup\left[v_{z}, v_{z}^{\prime}\right]$. Since $f\left(v_{y}\right)=f\left(v_{y}^{\prime}\right)=y$ and $f\left(v_{z}\right)=f\left(v_{z}^{\prime}\right)=z$, the assumptions on $f$ imply that $f\left(\left[v_{y}^{\prime}, v_{y}\right]\right)$ is contained in the $M_{2}$-ball around $y$ and $f\left(\left[v_{z}, v_{z}^{\prime}\right]\right)$ is contained in the $M_{2}$-ball around $z$ in $Y$. Moreover, we have already shown that $f\left(\left[v_{y}, v_{z}\right]\right)$ is $H^{\prime}$-Hausdorff close to $[y, z]$. Therefore $f\left(\left[v_{y}^{\prime}, v_{z}^{\prime}\right]\right)$ is contained in the $H$-neighborhood of $[y, z]=\left[f\left(v_{y}^{\prime}\right), f\left(y_{z}^{\prime}\right)\right]$ with $H=2 L \delta_{0}+M_{2}+H^{\prime}$. A similar argument shows that $[y, z]=\left[f\left(v_{y}^{\prime}\right), f\left(y_{z}^{\prime}\right)\right]$ is contained in the $H$-neighborhood of $f\left(\left[v_{y}^{\prime}, v_{z}^{\prime}\right]\right)$. Thus $[y, z]=\left[f\left(v_{y}^{\prime}\right), f\left(v_{z}^{\prime}\right)\right]$ and $f\left(\left[v_{y}^{\prime}, v_{z}^{\prime}\right]\right)$ are $H$-Hausdorff close, as required.

It is also possible to derive Corollary 2.4 using Proposition 3.1 from a new paper of Bowditch (December 2012, [9]) instead of Bowditch's hyperbolicity criterion from [8]. Proposition 3.1 of [9] gives a strengthened form of Theorem 3.11 of MasurSchleimer [25]. Theorem 3.11 of [25] is sufficient to conclude that the graph $Y$ in Corollary 2.4 is Gromov-hyperbolic; however, the existence of the constant $H$ as in the conclusion of Corollary 2.4 requires Proposition 3.1 of [9].

Proposition 2.5. For any positive integers $\delta_{0}, L, M$ and $D$ there exist $\delta_{1}>0$ and $H>0$ so that the following holds.

Let $X, Y$ be connected graphs, such that $X$ is $\delta_{0}$-hyperbolic. Let $f: X \rightarrow Y$ be a L-Lipschitz graph map for some $L \geq 0$. Let $S \subseteq V(X)$ be such that the following holds.

(1) We have $f(S)=V(Y)$.

(2) The set $S$ is $D$-dense in $X$ for some $D>0$.

(3) For $x, y \in S$, if $d(f(x), f(y)) \leq 1$ then for any geodesic $[x, y]$ in $X$ we have

$$
\operatorname{diam}_{Y}(f([x, y])) \leq M .
$$

Then $Y$ is $\delta_{1}$-hyperbolic and, for any $x, y \in V(X)$ and any geodesic $[x, y]$ in $X$, the path $f([x, y])$ is $H$-Hausdorff close to any geodesic $[f(x), f(y)]$ in $Y$. 
Proof. First we show that, for every $m_{1}>0$, there is $m_{2}>0$ so that whenever $x, y \in S$ satisfy $d_{Y}(f(x), f(y)) \leq m_{1}$ then $\operatorname{diam}_{Y}(f([x, y])) \leq m_{2}$. Indeed let $x, y \in S$ be as above and consider a geodesic path $[f(x), f(y)]$ in $Y$. Let

$$
f(x)=z_{0}, z_{1}, \ldots, z_{t}=f(y), \quad t<m_{1},
$$

be the sequence of consecutive vertices on $[f(x), f(y)]$. Let $x_{0}=x, x_{t}=y$ and for $1 \leq i \leq t-1$ let $x_{i} \in S$ be such that $f\left(x_{i}\right)=z_{i}$. Such $x_{i}$ exist since by assumption $f(S)=V(Y)$. We have $\operatorname{diam}_{Y} f\left(\left[x_{i}, x_{i+1}\right]\right) \leq M$. By hyperbolicity, the geodesic $[x, y]$ is contained in the $\left(m_{1} \delta_{0}\right)$-neighborhood of the union

$$
\bigcup_{i=0}^{t-1}\left[x_{i}, x_{i+1}\right] \text {. }
$$

Since $f$ is $L$-Lipschitz, $f([x, y])$ is contained in the $\left(L m_{1} \delta_{0}\right)$-neighborhood of

$$
\bigcup_{i=0}^{t-1} f\left(\left[x_{i}, x_{i+1}\right]\right) \text {. }
$$

But each $f\left(\left[x_{i}, x_{i+1}\right]\right)$ has diameter $\leq M$. Therefore, $f([x, y])$ has a diameter of at most $m_{2}=\left(m_{1} M+2 L m_{1} \delta_{0}\right)$.

Now let $M_{1} \geq 0$ and $x, y \in V(X)$ be arbitrary vertices with $d_{Y}(f(x), f(y)) \leq$ $M_{1}$. Since $S$ is $D$-dense in $X$, there exist $x^{\prime}, y^{\prime} \in S$ such that $d\left(x, x^{\prime}\right), d\left(y, y^{\prime}\right) \leq D$. The fact that $f$ is $L$-Lipschitz implies that $d\left(f\left(x^{\prime}\right), f\left(y^{\prime}\right)\right) \leq M_{1}+2 D L$. Therefore, by the above claim, it follows that the

$$
\operatorname{diam}_{Y} f\left(\left[x^{\prime}, y^{\prime}\right]\right) \leq m_{2}\left(M_{1}+2 D L\right) .
$$

Since $X$ is $\delta_{0}$-hyperbolic and $d\left(x, x^{\prime}\right), d\left(y, y^{\prime}\right) \leq D$, we have that $[x, y]$ and $\left[x^{\prime}, y^{\prime}\right]$ are $(2 \delta+2 D)$-Hausdorff close. Again, using that $f$ is $L$-Lipschitz, we conclude that $f([x, y])$ has a diameter of at most

$$
M_{2}=m_{2}\left(M_{1}+2 D L\right)+4 L\left(2 \delta_{0}+2 D\right) .
$$

The assumption of Corollary 2.4 are now satisfied for constants $\delta_{0}, L, M_{1}$ and $M_{2}$. Proposition 2.5 now follows from Corollary 2.4.

Proposition 2.5 easily implies the well-known fact that "coning-off" or "electrifying" a family of uniformly quasiconvex subsets in a hyperbolic space produces a hyperbolic space. Various versions of this statement have multiple appearances in the literature; see, for example, Lemma 4.5 and Proposition 4.6 in [13], Proposition 7.12 in [7], Lemma 2.3 in [28], Theorem 3.4 in [12], etc. Maher and Schleimer [22] appear to be the first to explicitly note that after "electrifying" a family of uniformly quasiconvex subsets in a hyperbolic space, not only is the resulting space again hyperbolic, but the image of a geodesic is a reparameterized quasigeodesic.

We give a version of the "coning-off" statement here phrased in the context of graphs with simplicial metrics. 
Proposition 2.6. Let $X$ be a connected graph with simplicial metric $d_{X}$ such that $\left(X, d_{X}\right)$ is $\delta_{0}$-hyperbolic. Let $C>0$ and $\left(X_{j}\right)_{j \in J}$ be a family of subgraphs of $X$ such that each $X_{j}$ is a $C$-quasiconvex subset of $X$. Let $Y$ be the graph obtained from $X$ by adding to $X$ the new edges $e_{x, y, j}$ with endpoints $x, y$ whenever $j \in J$ and $x$, $y$ are vertices of $X_{j}$ (thus $X$ is a subgraph of $Y$ ). Let $d_{Y}$ be the simplicial metric on $Y$.

Then $Y$ is $\delta_{1}$-hyperbolic for some constant $\delta_{1}>0$ depending only on $C$ and $\delta_{0}$. Moreover there exists $H=H\left(C, \delta_{0}\right)>0$ such that whenever $x, y \in V(X),[x, y]_{X}$ is a $d_{X}$-geodesic from $x$ to $y$ in $X$ and $[x, y]_{Y}$ is a $d_{Y}$-geodesic from $x$ to $y$ in $Y$ then $[x, y]_{X}$ and $[x, y]_{Y}$ are $H$-Hausdorff close in $\left(Y, d_{Y}\right)$.

Proof. Let $f: X \rightarrow Y$ be the inclusion map and put $S=V(X)$. We claim that the conditions of Proposition 2.5 are satisfied.

By construction $V(X)=V(Y)$, so $f(S)=V(Y)$. Also, the map $f$ is obviously 1-Lipschitz. Suppose now that $x, y \in V(X)$ are such that $d_{Y}(x, y) \leq 1$. If $d_{Y}(x, y)<1$ then $d_{Y}(x, y)=0$, so that $x=y$. In this case it is obvious that condition (3) of Proposition 2.5 holds for $f\left([x, y]_{X}\right)$. Suppose now that $d_{Y}(x, y)=1$. Thus there exists an edge $e$ in $Y$ with endpoints $x, y$. If $e$ is an edge of $X$ then $d_{X}(x, y)=1$ and it is again obvious that condition (3) of Proposition 2.5 holds for $f\left([x, y]_{X}\right)$. Suppose now that $e=e_{x, y, j}$ for some $j \in J$. Thus $x, y \in V\left(X_{j}\right)$. Since $X_{j}$ is $C$-quasiconvex in $X$, we see that for any point $u$ on $[x, y]_{X}$ there exists a vertex $z$ of $X_{j}$ with $d_{X}(u, z) \leq C$. Hence $d_{Y}(u, z) \leq C$ as well. We have $d_{Y}(x, z) \leq 1$ and $d_{Y}(z, y) \leq 1$ since $x, y, z$ are vertices of $X_{j}$. Hence $d_{Y}(f(u), x)=d_{Y}(u, x) \leq C+1$. Thus $f\left([x, y]_{X}\right)$ is contained in the $d_{Y}$-ball of radius $C+1$ centered at $x$ in $\left(Y, d_{Y}\right)$, and again condition (3) of Proposition 2.5 holds.

Thus Proposition 2.5 applies and the conclusion of Proposition 2.6 follows.

Note that the assumptions of Proposition 2.6 do not require the family $\left(X_{j}\right)_{j \in J}$ to be "sufficiently separated". Such a requirement is present in many versions of Proposition 2.6 available in the literature, although this assumption is not in fact necessary and, in particular, Proposition 7.12 in [7] does not impose the "sufficiently separated" requirement. We have derived Proposition 2.6 from Proposition 2.5, which in turn was a consequence of Corollary 2.4. A close comparison of these statements show that the converse implication does not work, and that Corollary 2.4 is a more general statement than Proposition 2.6.

\section{Free factor complex and free splitting complex}

Definition 3.1 (Free factor complex). Let $F_{N}$ be a free group of finite rank $N \geq 3$. The free factor complex $\mathrm{FF}_{N}$ of $F_{N}$ is a simplicial complex defined as follows. The set of vertices $V\left(\mathrm{FF}_{N}\right)$ of $\mathrm{FF}_{N}$ is defined as the set of all $F_{N}$-conjugacy classes 
$[A]$ of proper free factors $A$ of $F_{N}$. Two distinct vertices $[A]$ and $[B]$ of $\mathrm{FF}_{N}$ are joined by an edge whenever there exist proper free factors $A, B$ of $F_{N}$ representing $[A]$ and $[B]$ respectively, such that either $A \leq B$ or $B \leq A$.

More generally, for $k \geq 1$, a collection of $k+1$ distinct vertices $\left[A_{0}\right], \ldots,\left[A_{k}\right]$ of $\mathrm{FF}_{N}$ spans a $k$-simplex in $\mathrm{FF}_{N}$ if, up to a possible re-ordering of these vertices there exist representatives $A_{i}$ of $\left[A_{i}\right]$ such that $A_{0} \leq A_{1} \leq \cdots \leq A_{k}$.

There is a canonical action of $\operatorname{Out}\left(F_{N}\right)$ on $\mathrm{FF}_{N}$ by simplicial automorphisms: If

$$
\Delta=\left\{\left[A_{0}\right], \ldots,\left[A_{k}\right]\right\}
$$

is a $k$ simplex and $\varphi \in \operatorname{Out}\left(F_{N}\right)$, then

$$
\varphi(\Delta):=\left\{\left[\varphi\left(A_{0}\right)\right], \ldots,\left[\varphi\left(A_{k}\right)\right]\right\} .
$$

It is not hard to check that for $N \geq 3$ the complex $\mathrm{FF}_{N}$ is connected, has dimension $N-2$ and that $\mathrm{FF}_{N} / \operatorname{Out}\left(F_{N}\right)$ is compact.

Definition 3.2 (Free splitting complex). Let $F_{N}$ be a free group of finite rank $N \geq 3$.

The free splitting complex $\mathrm{FS}_{N}$ is a simplicial complex defined as follows. The vertex set $V\left(\mathrm{FS}_{N}\right)$ consists of equivalence classes of splittings $F_{N}=\pi_{1}(\mathbb{A})$, where $\mathbb{A}$ is a graph of groups with a single topological edge $e$ (possibly a loop edge) and the trivial edge group such that the action of $F_{N}$ on the Bass-Serre tree $T_{\mathbb{A}}$ is minimal (i.e. such that if $e$ is a non-loop edge then both vertex groups in $\mathbb{A}$ are nontrivial). Two such splittings $F_{N}=\pi_{1}(\mathbb{A})$ and $F_{N}=\pi_{1}(\mathbb{B})$ are equivalent if there exists an $F_{N}$-equivariant isometry between $T_{\mathbb{A}}$ and $T_{\mathbb{B}}$. We denote the equivalence class of a splitting $F_{N}=\pi_{1}(\mathbb{A})$ by $[\mathbb{A}]$.

The edges in $\mathrm{FS}_{N}$ correspond to two splittings admitting a common refinement. Thus two distinct vertices $[\mathbb{A}]$ and $[\mathbb{B}]$ of $\mathrm{FS}_{N}$ are joined by an edge whenever there exists a splitting $F_{N}=\pi_{1}(\mathbb{D})$ such that the graph of groups $\mathbb{D}$ has exactly two topological edges, both with trivial edge groups, and such that collapsing one of these edges produces a splitting of $F_{N}$ representing $[\mathbb{A}]$ and collapsing the other edge produces a splitting representing $[\mathbb{B}]$.

More generally, for $k \geq 1$ a collection of $k+1$ distinct vertices $\left[\mathbb{A}_{0}\right], \ldots,\left[\mathbb{A}_{k}\right]$ of $\mathrm{FS}_{N}$ spans a $k$-simplex in $\mathrm{FS}_{N}$ whenever there exists a splitting $F_{N}=\pi_{1}(\mathbb{D})$ such that the graph of groups $\mathbb{D}$ has the following properties:

(a) The underlying graph of $\mathbb{D}$ has exactly $k+1$ topological edges, $e_{0}, \ldots, e_{k}$.

(b) The edge group of each $e_{i}$ is trivial.

(c) For each $i=0, \ldots, k$ collapsing all edges except for $e_{i}$ in $\mathbb{D}$ produces a splitting of $F_{N}$ representing $\left[\mathbb{A}_{i}\right]$.

The complex $\mathrm{FS}_{N}$ comes equipped with a natural action of $\operatorname{Out}\left(F_{N}\right)$ by simplicial automorphisms. 
Again, it is not hard to check that for $N \geq 3$ the complex $\mathrm{FS}_{N}$ is finite-dimensional, connected and that the quotient $\mathrm{FS}_{N} / F_{N}$ is compact.

We denote the barycentric subdivision of $\mathrm{FS}_{N}$ by $\mathrm{FS}_{N}^{\prime}$.

Definition 3.3 (Marking). Let $N \geq 2$. Recall that a marking on $F_{N}$ is an isomorphism $\alpha: F_{N} \rightarrow \pi_{1}(\Gamma, v)$ where $\Gamma$ is a finite connected graph without any degree-one and degree-two vertices and $v$ is a vertex of $\Gamma$. By abuse of notation, if $\alpha$ is specified, we will often refer to $\Gamma$ as a marking.

Two markings $\alpha: F_{N} \rightarrow \pi_{1}(\Gamma, v)$ and $\alpha^{\prime}: F_{N} \rightarrow \pi_{1}\left(\Gamma^{\prime}, v^{\prime}\right)$ are said to be equivalent, if there exists an $F_{N}$-equivariant isometry $\widetilde{(\Gamma, v)} \rightarrow \widetilde{\left(\Gamma^{\prime}, v^{\prime}\right)}$. The equivalence class of a marking $\alpha: F_{N} \rightarrow \pi_{1}(\Gamma, v)$ is denoted by $[\alpha]$ or, if $\alpha$ is already specified, just $[\Gamma]$.

Convention 3.4 (Barycenters). Note that for $N \geq 3$ any marking $\alpha: F_{N} \rightarrow \pi_{1}(\Gamma)$ corresponds to a simplex $\Delta_{\alpha}$ in $\mathrm{FS}_{N}$, as follows. We can view $\Gamma$ as a graph of groups by assigning trivial groups to all the vertices and edges of $\Gamma$. Then the vertices of $\Delta_{\alpha}$ correspond to the (topological) edges of $\Gamma$ and come from choosing an edge $e$ of $\Gamma$ and collapsing all the other edges of $\Gamma$. It is easy to see that $\Delta_{\alpha}$ depends only on the equivalence class $[\alpha]$ of the marking $\alpha$.

We denote the vertex of $\mathrm{FS}_{N}^{\prime}$ given by the barycenter of $\Delta_{\alpha}$ by $z(\alpha)$ or, if it is more convenient, by $z(\Gamma)$. Note that if $[\alpha]=[\beta]$ then $z(\alpha)=z(\beta)$. We will sometimes refer to a marking $\Gamma$ as a vertex of $\mathrm{FS}_{N}^{\prime}$; when that happens, we always mean the vertex $z(\Gamma)$.

\section{The free bases graph}

If $\Gamma$ is a graph (i.e. a one-dimensional $\mathrm{CW}$-complex), then any topological edge (i.e. a closed 1-cell) of $\Gamma$ is homeomorphic to either $[0,1]$ or to $\mathbb{S}^{1}$ and thus admits exactly two orientations. An oriented edge of $\Gamma$ is a topological edge together with a choice of an orientation on this edge. If $e$ is an oriented edge of $\Gamma$, we denote by $e^{-1}$ the oriented edge obtained by changing the orientation on $e$ to the opposite one. Note that $\left(e^{-1}\right)^{-1}=e$ for any oriented edge $e$. For an oriented edge $e$ we denote the initial vertex of $e$ by $o(e)$ and the terminal vertex of $e$ by $t(e)$. Then $o\left(e^{-1}\right)=t(e)$ and $t\left(e^{-1}\right)=o(e)$. We will denote by $E \Gamma$ the set of oriented edges of $\Gamma$ and by $V \Gamma$ the set of vertices of $\Gamma$.

Let $N \geq 2$. We denote by $W_{N}$ the graph with a single vertex $v_{0}$ and $N$ distinct oriented loop-edges $e_{1}, \ldots, e_{N}$.

Definition 4.1 ( $\mathcal{A}$-rose). Let $\mathcal{A}=\left\{a_{1}, \ldots, a_{N}\right\}$ be a free basis of $F_{N}$. Define the $\mathcal{A}$-rose $R_{\mathcal{A}}$ as the marking $\alpha_{\mathcal{A}}: F_{N} \rightarrow \pi_{1}\left(W_{N}, v_{0}\right)$ where $\alpha_{\mathcal{A}}$ sends $a_{i}$ to the loop at $v_{0}$ in $\Gamma$ corresponding to $e_{i}$, traversed in the direction given by the orientation of $e_{i}$. 
Definition 4.2 (Free bases graph). Let $N \geq 3$. The free bases graph $\mathrm{FB}_{N}$ of $F_{N}$ is a simple graph defined as follows. The vertex set $V\left(\mathrm{FB}_{N}\right)$ consists of equivalence classes free bases $\mathcal{A}$ of $F_{N}$. Two free bases $\mathcal{A}$ and $\mathcal{B}$ of $F_{N}$ are considered equivalent if the Cayley graphs $T_{\mathscr{A}}$ and $T_{\mathscr{B}}$ of $F_{N}$ with respect to $\mathcal{A}$ and $\mathscr{B}$ are $F_{N}$-equivariantly isometric. We denote the equivalence class of a free basis $\mathcal{A}$ of $F_{N}$ by [A].

Note that for free bases $\mathcal{A}=\left\{a_{1}, \ldots, a_{N}\right\}$ and $\mathcal{B}=\left\{b_{1}, \ldots, b_{N}\right\}$ of $F_{N}$ we have $[\mathcal{A}]=[\mathscr{B}]$ if and only if there exist a permutation $\sigma \in S_{N}$, an element $g \in F_{N}$ and numbers $\varepsilon_{i} \in\{1,-1\}$ (where $i=1, \ldots, N$ ) such that

$$
b_{i}=g^{-1} a_{\sigma(i)}^{\varepsilon_{i}} g
$$

for $i=1, \ldots, N$. Thus $[\mathcal{A}]=[\mathscr{B}]$ if and only if the roses $R_{\mathscr{A}}$ and $R_{\mathscr{B}}$ are equivalent as markings. Note also that for any free basis $\mathcal{A}$ of $F_{N}$ and any $g \in F_{N}$ we have $\left[g^{-1} \mathcal{A} g\right]=[\mathcal{A}]$.

The edges in $\mathrm{FB}_{N}$ are defined as follows. Let $[\mathcal{A}]$ and $[\mathscr{B}]$ be two distinct vertices of $\mathrm{FB}_{N}$. These vertices are adjacent in $\mathrm{FB}_{N}$ whenever there exists $a \in \mathcal{A}$ such that some element $b \in \mathscr{B}$ is conjugate to $a$ or $a^{-1}$. Thus two distinct vertices $v_{1}, v_{2}$ of $\mathrm{FB}_{N}$ are adjacent if and only if there exist free bases $\mathcal{A}$ and $\mathcal{B}$ representing $v_{1}, v_{2}$ accordingly such that $\mathcal{A} \cap \mathscr{B} \neq \varnothing$.

The graph $\mathrm{FB}_{N}$ comes equipped with a natural $\operatorname{Out}\left(F_{N}\right)$ action by simplicial automorphisms.

Proposition 4.3. Let $N \geq 3$. Then the following holds.

(1) The graph $\mathrm{FB}_{N}$ is connected.

(2) For each vertex $v=[\mathcal{A}]$ of $\mathrm{FB}_{N}$ choose some $a_{v} \in \mathcal{A}$. Consider the map

$$
h: V\left(\mathrm{FB}_{N}\right) \rightarrow V\left(\mathrm{FF}_{N}\right)
$$

defined as $h(v)=\left[\left\langle a_{v}\right\rangle\right]$ for every vertex $v$ of $\mathrm{FB}_{N}$. Extend $h$ to a graph-map

$$
h: \mathrm{FB}_{N} \rightarrow \mathrm{FF}_{N}
$$

by sending every edge e of $\mathrm{FB}_{N}$ with endpoints $v, v^{\prime}$ to a geodesic path $\left[h(v), h\left(v^{\prime}\right)\right]$ in $\mathrm{FF}_{N}^{(1)}$. Then:

(a) The map $h$ is a quasi-isometry. In particular, the complexes $\mathrm{FB}_{N}$ and $\mathrm{FF}_{N}$ are quasi-isometric.

(b) The set $h\left(V\left(\mathrm{FB}_{N}\right)\right)$ is 3-dense in $\mathrm{FF}_{N}^{(1)}$

Proof. First we will show that $h$ is 4-Lipschitz. Since $h: \mathrm{FB}_{N} \rightarrow \mathrm{FF}_{N}^{(1)}$ is a graphmap, it suffices to check that for any two adjacent vertices $v, v^{\prime}$ of $\mathrm{FB}_{B}$ we have $d_{\mathrm{FF}_{N}}\left(h(v), h\left(v^{\prime}\right)\right) \leq 4$.

Let $v=[\mathcal{A}]$ and $v^{\prime}=[\mathscr{B}]$ be two adjacent vertices of $\mathrm{FB}_{N}$. Hence we may choose free bases $\mathcal{A}$ representing $v$ and $\mathscr{B}$ representing $v^{\prime}$ such that $\mathcal{A} \cap \mathscr{B} \neq \emptyset$. Up to reordering these bases, we may assume that $\mathcal{A}=\left\{a_{1}, \ldots, a_{N}\right\}, \mathcal{B}=\left\{b_{1}, \ldots, b_{N}\right\}$ 
and that $a_{1}=b_{1}$. Then $a_{v}=a_{i}$ and $a_{v^{\prime}}=b_{j}$ for some $1 \leq i, j \leq N$ and thus, by definition of $h$, we have $h(v)=\left[\left\langle a_{i}\right\rangle\right], h\left(v^{\prime}\right)=\left[\left\langle b_{j}\right\rangle\right]$.

We will assume that $i>1$ and $j>1$ as the cases where $i=1$ or $j=1$ are easier. Then in $\mathrm{FF}_{N}$ we have

$$
d_{\mathrm{FF}_{N}}\left(\left[\left\langle a_{i}\right\rangle\right],\left[\left\langle a_{i}, a_{1}\right\rangle\right]\right)=d_{\mathrm{FF}_{N}}\left(\left[\left\langle a_{i}, a_{1}\right\rangle\right],\left[\left\langle a_{1}\right\rangle\right]\right)=1
$$

and

$$
d_{\mathrm{FF}_{N}}\left(\left[\left\langle b_{j}\right\rangle\right],\left[\left\langle b_{j}, b_{1}\right\rangle\right]\right)=d_{\mathrm{FF}_{N}}\left(\left[\left\langle b_{j}, b_{1}\right\rangle\right],\left[\left\langle b_{1}\right\rangle\right]\right)=1 .
$$

Since $a_{1}=b_{1}$, by the triangle inequality we conclude that

$$
d_{\mathrm{FF}_{N}}\left(h(v), h\left(v^{\prime}\right)\right)=d_{\mathrm{FF}_{N}}\left(\left[\left\langle a_{i}\right\rangle\right],\left[\left\langle b_{j}\right\rangle\right]\right) \leq 4 .
$$

Thus the map $h$ is 4-Lipschitz, as claimed.

To show that $h$ is a quasi-isometry we will construct a "quasi-inverse", that is a Lipschitz map $q: \mathrm{FF}_{N}^{(1)} \rightarrow \mathrm{FB}_{N}$ such that there exists $C \geq 0$ with the property that for every vertex $v$ of $\mathrm{FB}_{N}, d_{\mathrm{FB}_{N}}(v, q(h(v))) \leq C$ and that for every vertex $u$ of $\mathrm{FF}_{N}, d_{\mathrm{FF}_{N}}(u, h(q(u))) \leq C$.

We define $q$ on $V\left(\mathrm{FF}_{N}\right)$ and then extend $q$ to edges in a natural way, by sending every edge to a geodesic joining the images of its end-vertices.

Let $u=[K]$ be an arbitrary vertex of $\mathrm{FF}_{N}$ (so that $K$ is a proper free factor of $\left.F_{N}\right)$. We choose a free basis $\mathcal{B}_{K}$ of $K$ and then a free basis $\mathcal{A}_{K}$ of $F_{N}$ such that $\mathcal{B}_{K} \subseteq \mathcal{A}_{K}$. Put $q(u)=\left[\mathcal{A}_{K}\right]$.

First we check that $q$ is Lipschitz. Let $u=[K]$ and $u^{\prime}=\left[K^{\prime}\right]$ be adjacent vertices of $\mathrm{FF}_{N}$, where $K, K^{\prime}$ are proper free factors of $F_{N}$. We may assume that $K \leq K^{\prime}$ is a proper free factor of $K^{\prime}$. Since $K^{\prime} \neq F_{N}$, there exists $t \in \mathcal{A}_{K^{\prime}} \backslash \mathcal{B}_{K^{\prime}}$. Since $K$ is a free factor of $K^{\prime}$, we can find a free basis $\mathcal{A}$ of $F_{N}$ such that $t \in \mathscr{A}$ and $\mathscr{B}_{K} \subseteq \mathcal{A}$. Since $t \in \mathcal{A}_{K^{\prime}} \cap \mathcal{A}$, we have $d\left(\left[\mathcal{A}_{K^{\prime}}\right],[\mathcal{A}]\right) \leq 1$ in $\mathrm{FB}_{N}$. Since $\mathscr{B}_{K} \subset \mathcal{A} \cap \mathcal{A}_{K}$, it follows that $d\left(\left[\mathcal{A}_{K}\right],[\mathcal{A}]\right) \leq 1$ in $\mathrm{FB}_{N}$. Therefore

$$
d_{\mathrm{FB}_{N}}\left(q(u), q\left(u^{\prime}\right)\right)=d_{\mathrm{FB}_{N}}\left(\left[\mathcal{A}_{K}\right],\left[\mathcal{A}_{K^{\prime}}\right]\right) \leq 2 .
$$

Hence $q$ is 2-Lipschitz.

For a vertex $v=[\mathcal{A}]$ of $\mathrm{FB}_{N}$ let us now estimate $d_{\mathrm{FB}_{N}}(v, q(h(v)))$. We have $h(v)=\left[\left\langle a_{v}\right\rangle\right]$ for some $a_{v} \in \mathcal{A}$. The group $K=\left\langle a_{v}\right\rangle$ is infinite cyclic (that is free of rank 1). Therefore this group has only two possible free bases, $\left\{a_{v}\right\}$ and $\left\{a_{v}^{-1}\right\}$. We will assume that $\mathscr{B}_{K}=\left\{a_{v}\right\}$ as the case $\mathscr{B}_{K}=\left\{a_{v}^{-1}\right\}$ is similar. Then, by definition, $\mathcal{A}_{K}$ is a free basis of $F_{N}$ containing $a_{v}$ and $q(h(v))=q([K])=\left[\mathcal{A}_{K}\right]$. Thus $a_{v} \in \mathcal{A} \cap \mathcal{A}_{K}$ and hence $d_{\mathrm{FB}_{N}}(v, q(h(v))) \leq 1$ in $\mathrm{FB}_{N}$.

Now let $u=[K]$ be an arbitrary vertex of $\mathrm{FF}_{N}$. We need to estimate the distance $d_{\mathrm{FF}_{N}}(u, h(q(u)))$. By definition, $v:=h(u)=\left[\mathcal{A}_{K}\right]$ where $\mathcal{A}_{K}$ is a free basis of $F_{N}$ containing as a (proper) subset a free basis $\mathscr{B}_{K}$ of $K$. Then $a_{v} \in \mathcal{A}_{K}$ and 
$h(q(u))=h(v)=\left[\left\langle a_{v}\right\rangle\right]$. Choose an element $b \in \mathscr{B}_{K}$. It may happen that $b=a_{v}$, but in any case $K^{\prime}:=\left\langle b, a_{v}\right\rangle$ is a proper free factor of $F_{N}$. Then

$$
d_{\mathrm{FF}_{N}}([K],[\langle b\rangle]) \leq 1, \quad d_{\mathrm{FF}_{N}}\left([\langle b\rangle],\left[K^{\prime}\right]\right) \leq 1 \quad \text { and } \quad d_{\mathrm{FF}_{N}}\left(\left[K^{\prime}\right],\left[\left\langle a_{v}\right\rangle\right]\right) \leq 1 .
$$

Therefore

$$
d_{\mathrm{FF}_{N}}\left(u, h(q(u))=d_{\mathrm{FF}_{N}}\left([K],\left[\left\langle a_{v}\right\rangle\right]\right) \leq 3 .\right.
$$

Thus indeed $q$ is a quasi-inverse for $h$, and hence $h$ is a quasi-isometry, as required.

We next show that $h\left(V\left(\mathrm{FB}_{N}\right)\right)$ is 3-dense in $\mathrm{FF}_{N}^{(1)}$. Indeed, let $K \leq F_{N}$ be an arbitrary proper free factor of $F_{N}$. Let $a_{1}, \ldots, a_{m}$ (where $1 \leq m<N$ ) be a free basis of $K$ and choose $a_{m+1} \ldots, a_{N}$ such that $\mathcal{A}=\left\{a_{1}, \ldots, a_{N}\right\}$ is a free basis of

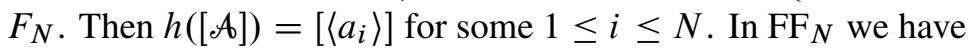

$$
\begin{aligned}
d_{\mathrm{FF}_{N}}\left([K],\left[\left\langle a_{1}\right\rangle\right]\right) & \leq 1, \\
d_{\mathrm{FF}_{N}}\left(\left[\left\langle a_{1}\right\rangle\right],\left[\left\langle a_{1}, a_{i}\right\rangle\right]\right) & \leq 1
\end{aligned}
$$

and

$$
d_{\mathrm{FF}_{N}}\left(\left[\left\langle a_{1}, a_{i}\right\rangle\right],\left[\left\langle a_{i}\right\rangle\right]\right) \leq 1 .
$$

Since $h([\mathcal{A}])=\left[\left\langle a_{i}\right\rangle\right]$, it follows that $d_{\mathrm{FF}_{N}}([K], h(v)) \leq 3$. Thus indeed $h\left(V\left(\mathrm{FB}_{N}\right)\right)$ is 3-dense in $\mathrm{FF}_{N}^{(1)}$, as claimed.

Definition 4.4 (Free basis defined by a marking). If $\alpha: F_{N} \rightarrow \pi_{1}(\Gamma, v)$ is a marking, and $T \subseteq \Gamma$ is a maximal tree in $\Gamma$, there is a naturally associated free basis $\mathscr{B}(\alpha, T)$ (which we will also sometimes denote $\mathcal{B}(\Gamma, T)$ ) of $F_{N}$. Namely, in this case $\Gamma-T$ consists of $N$ topological edges. Choose oriented edges $e_{1}, \ldots, e_{N} \in E(\Gamma-T)$ so that $E(\Gamma-T)=\left\{e_{1}^{ \pm 1}, \ldots, e_{N}^{ \pm 1}\right\}$. For $j=1, \ldots, N$ put

$$
\gamma_{j}=\left[v, o\left(e_{j}\right)\right]_{T} e_{j}\left[t\left(e_{j}\right), v\right]_{T},
$$

where $\left[u, u^{\prime}\right]_{T}$ denotes the (unique) geodesic in the tree $T$ from $u$ to $u^{\prime}$ for $u, u^{\prime} \in$ $V(\Gamma)=V(T)$.

Then $\gamma_{1}, \ldots, \gamma_{N}$ is a free basis of $\pi_{1}(\Gamma, v)$. Put

$$
\mathscr{B}(\alpha, T):=\left\{\alpha^{-1}\left(\gamma_{1}\right), \ldots, \alpha^{-1}\left(\gamma_{1}\right)\right\} .
$$

Remark 4.5. One can show that there is a constant $C=C(N)>0$ such that if $\alpha: F_{N} \rightarrow \pi_{1}(\Gamma, v)$ and $\alpha^{\prime}: F_{N} \rightarrow \pi_{1}\left(\Gamma^{\prime}, v^{\prime}\right)$ are equivalent markings and $T \subseteq \Gamma$, $T^{\prime} \subseteq \Gamma^{\prime}$ are maximal trees, then

$$
d_{\mathrm{FB}_{N}}\left([\mathcal{B}(\alpha, T)],\left[\mathcal{B}\left(\alpha^{\prime}, T^{\prime}\right)\right]\right) \leq C .
$$

This can be shown, for example, using the quasi-isometry $q: \mathrm{FB}_{N} \rightarrow \mathrm{FF}_{N}$ constructed in Proposition 4.3. Thus the definitions imply that if $T$ is a maximal tree in $\Gamma$ 
and $e$ is an edge of $\Gamma \backslash T$, then $q([\mathcal{B}(\alpha, T)])$ is a bounded distance away in $\mathrm{FF}_{N}$ from the free factor of $F_{N}$ corresponding to any of the vertex groups in the graph of groups $\Gamma_{e}$ obtained by collapsing $\Gamma \backslash e$. On the other hand, for any two edges $e_{1}, e_{2}$ of $\Gamma$ the free splittings $\Gamma_{e_{1}}$ and $\Gamma_{e_{2}}$ are adjacent vertices of $\mathrm{FS}_{N}$ and therefore (e.g. using the Lipschitz map $\tau: \mathrm{FS}_{N} \rightarrow \mathrm{FF}_{N}$ from the Introduction), any two vertex groups $A_{1}$ and $A_{2}$ from these splittings are bounded distance away in $\mathrm{FF}_{N}$.

\section{A-graphs and Stallings folds}

We briefly discuss here the language and machinery of Stallings foldings, introduced by Stallings in a seminal paper [31]. We refer the reader to [21] for detailed background on the topic.

If $\Gamma$ is a finite connected non-contractible graph, we denote by $\operatorname{Core}(\Gamma)$ the unique minimal subgraph of $\Gamma$ such that the inclusion $\operatorname{Core}(\Gamma) \subseteq \Gamma$ is a homotopy equivalence. Thus Core $(\Gamma)$ carries $\pi_{1}(\Gamma)$ and we can obtain $\Gamma$ from Core $(\Gamma)$ by attaching finitely many trees.

Definition 5.1 ( $\mathcal{A}$-graph). Let $\mathcal{A}$ be a free basis of $F_{N}$ and let $R_{\mathscr{A}}$ be the corresponding rose marking. An $\mathcal{A}$-graph is a graph $\Gamma$ with a labelling function $\mu: E \Gamma \rightarrow \mathcal{A}^{ \pm 1}$ (where $E \Gamma$ is the set of oriented edges of $\Gamma$ ) such that for every oriented edge $e \in E \Gamma$ we have $\mu\left(e^{-1}\right)=\mu(e)^{-1}$.

Note that there is an obvious way to view the rose $R_{\mathscr{A}}$ as an $\mathcal{A}$-graph. Any $\mathcal{A}$ graph $\Gamma$ comes equipped with a canonical label-preserving graph-map $p: \Gamma \rightarrow R_{\mathcal{A}}$ which sends all vertices of $\Gamma$ to the (unique) vertex of $R_{\mathscr{A}}$ and which sends every oriented edge of $\Gamma$ to the oriented edge of the rose $R_{\mathcal{A}}$ with the same label. We call $p$ the natural projection.

Let $\Gamma$ be a finite connected $\mathcal{A}$-graph containing at least one vertex of degree $\geq 3$. Following Handel-Mosher [16], we call vertices of $\Gamma$ that have degree $\geq 3$ natural vertices. The complement of the set of natural vertices in $\Gamma$ consists of a disjoint union of intervals whose closures, again following [16], we call natural edges.

Recall that in the definition of a marking on $F_{N}$ the graph appearing in that definition had no degree-one and degree-two vertices.

Remark 5.2. Suppose that $\Gamma$ is a connected $\mathcal{A}$-graph such that the natural projection $\Gamma \rightarrow R_{\mathcal{A}}$ is a homotopy equivalence. Then the projection $p: \operatorname{Core}(\Gamma) \rightarrow R_{\mathcal{A}}$ is a homotopy equivalence.

Then, via using the homotopy inverse of $p$ and making inverse subdivisions in $\operatorname{Core}(\Gamma)$ to erase all the degree-2 vertices, we get an actual marking of $F_{N}$, $\alpha: F_{N} \rightarrow \pi_{1}(\bar{\Gamma})$. Here $\bar{\Gamma}$ is the graph obtained from Core $(\Gamma)$ by doing inverse edge-subdivisions to erase all degree-two vertices. In this case we call $\alpha$ the marking associated with $\Gamma$ and denote $\alpha$ by $\alpha_{\Gamma}$, or, sometimes just by $\bar{\Gamma}$. 
Definition 5.3 (Folded graphs and Stallings folds). Let $\Gamma$ be an $\mathcal{A}$-graph. We say that $\Gamma$ is folded if there does not exist a vertex $v$ of $\Gamma$ and two distinct oriented edges $e_{1}, e_{2}$ with $o\left(e_{1}\right)=o\left(e_{2}\right)=v$ such that $\mu\left(e_{1}\right)=\mu\left(e_{2}\right)$. Otherwise we say that $\Gamma$ is non-folded.

Let $\Gamma$ be a non-folded $\mathcal{A}$-graph, let $e_{1}, e_{2}$ be two distinct oriented edges of $\Gamma$ such that $o\left(e_{1}\right)=o\left(e_{2}\right)=v \in V(\Gamma)$ and such that $\mu\left(e_{1}\right)=\mu\left(e_{2}\right)=a \in \mathcal{A}^{ \pm 1}$. Construct an $\mathcal{A}$-graph $\Gamma^{\prime}$ by identifying the edges $e_{1}$ and $e_{2}$ into a single edge $e$ with label $\mu(e)=a$. We say that $\Gamma^{\prime}$ is obtained from $\Gamma$ by a Stallings fold. In this case there is also a natural label-preserving fold map $f: \Gamma \rightarrow \Gamma^{\prime}$. It is easy to see that the fold map $f$ is a homotopy equivalence if and only if $t\left(e_{1}\right) \neq t\left(e_{2}\right)$ in $\Gamma$. If $t\left(e_{1}\right) \neq t\left(e_{2}\right)$ in $\Gamma$, we say that $f$ is a type-I Stallings fold. If $t\left(e_{1}\right)=t\left(e_{2}\right)$ in $\Gamma$, we say that $f$ is a type-II Stallings fold.

Note that if $\Gamma$ is a finite connected $\mathcal{A}$-graph such that the natural projection $\Gamma \rightarrow R_{\mathcal{A}}$ is a homotopy equivalence, and if $\Gamma^{\prime}$ is obtained from $\Gamma$ by a Stallings fold $f$, then $f$ is necessarily a type-I fold, and hence the natural projection $\Gamma^{\prime} \rightarrow R_{\mathcal{A}}$ is again a homotopy equivalence.

Definition 5.4 (Maximal fold). Let $\Gamma$ be a non-folded finite connected $\mathcal{A}$-graph, let $v \in V A$ be a natural vertex, let $e_{1}, e_{2}$ be two distinct oriented edges of $\Gamma$ such that $o\left(e_{1}\right)=o\left(e_{2}\right)=v$ and such that $\mu\left(e_{1}\right)=\mu\left(e_{2}\right)=a \in \mathcal{A}^{ \pm 1}$. Let $\widehat{e_{1}}$ and $\widehat{e_{2}}$ be the natural edges in $\Gamma$ that begin with $e_{1}, e_{2}$ accordingly. Let $z_{1}, z_{2}$ be maximal initial segments of $\widehat{e_{1}}$ and $\widehat{e_{2}}$ such that the label $\mu\left(z_{1}\right)$ is graphically equal, as a word over $\mathcal{A}^{ \pm 1}$, to the label $\mu\left(z_{2}\right)$. Thus $z_{1}$ starts with $e_{1}$ and $z_{2}$ starts with $e_{2}$. Let $\Gamma^{\prime}$ be obtained from $\Gamma$ by a chain of Stallings folds that fold $z_{1}$ and $z_{2}$ together. We say that $\Gamma^{\prime}$ is obtained from $\Gamma$ by a maximal fold. Being a composition of several Stallings folds, a maximal fold also comes equipped with a fold map $f: \Gamma \rightarrow \Gamma^{\prime}$.

Remark 5.5. Let $\Gamma$ be a connected $\mathcal{A}$-graph such that $\Gamma=\operatorname{Core}(\Gamma)$ and such that the natural projection $p: \Gamma \rightarrow R_{\mathcal{A}}$ is a homotopy equivalence. Let $\alpha: F_{N} \rightarrow \pi_{1}(\Gamma, v)$ be an associated marking. Let $T \subseteq \Gamma$ be a maximal tree.

Recall that according to Definition 4.4, we have an associated free basis $\mathcal{B}(\Gamma, T)$ of $F_{N}$. In this case $\mathcal{B}(\Gamma, T)$ can be described more explicitly as follows. Choose oriented edges $e_{1}, \ldots, e_{N} \in E(\Gamma-T)$ so that $E(\Gamma-T)=\left\{e_{1}^{ \pm 1}, \ldots, e_{N}^{ \pm 1}\right\}$. Let $w_{j}$ be the label (i.e. a word over $\mathcal{A}$ ) of the path $\left[v, o\left(e_{j}\right)\right]_{T} e_{j}\left[t\left(e_{j}\right), v\right]_{T}, j=1, \ldots, N$. Then $\mathcal{B}(\Gamma, T)=\left\{w_{1}, \ldots, w_{N}\right\}$.

We need the following technical notion which is a variant of the notion of a foldable map from the paper of Handel-Mosher [16].

Definition 5.6 (Foldable maps). Let $\Gamma$ be a finite connected $\mathcal{A}$-graph such that the natural projection $p: \Gamma \rightarrow R_{\mathcal{A}}$ is a homotopy equivalence and such that $\Gamma=\operatorname{Core}(\Gamma)$.

We say that the natural projection $p: \Gamma \rightarrow R_{\mathcal{A}}$ is foldable if the following conditions hold: 
(1) If $v$ is a vertex of degree 2 in $\Gamma$ and $e_{1}, e_{2}$ are the two distinct edges in $\Gamma$ with $o\left(e_{1}\right)=o\left(e_{2}\right)=v$ then $\mu\left(e_{1}\right) \neq \mu\left(e_{2}\right)$.

(2) If $\operatorname{deg}(v) \geq 3$ in $\Gamma$ then there exist three (oriented) edges $e_{1}, e_{2}, e_{3}$ in $\Gamma$ such that $o\left(e_{1}\right)=o\left(e_{2}\right)=o\left(e_{3}\right)=v$ and such that $\mu\left(e_{1}\right), \mu\left(e_{2}\right), \mu\left(e_{3}\right)$ are three distinct elements in $\mathcal{A}^{ \pm 1}$.

If the natural projection $p: \Gamma \rightarrow R_{\mathscr{A}}$ is foldable, we will also sometimes say that the $\mathcal{A}$-graph $\Gamma$ is foldable.

Remark 5.7. Let $\Gamma$ be a foldable $\mathcal{A}$-graph and let $\Gamma^{\prime}$ be obtained from $\Gamma$ by a maximal fold.

(1) One can check that $\Gamma^{\prime}$ is again foldable. Note, however, that a single Stallings fold on a foldable $\mathcal{A}$-graph may introduce a vertex of degree three where condition (2) of Definition 5.6 fails, so that the resulting graph is not foldable. Performing maximal folds instead of single Stallings folds avoids this problem.

(2) Lemma 2.5 in [16] implies that

$$
d_{\mathrm{FS}_{N}^{\prime}}\left(z(\bar{\Gamma}), z\left(\overline{\Gamma^{\prime}}\right)\right) \leq 2 .
$$

Recall that the marking $\bar{\Gamma}$ was defined in Remark 5.2.

(3) As noted above, in [16] Handel and Mosher introduce the notion of a "foldable" $F_{N}$-equivariant map between trees corresponding to arbitrary minimal splittings of $F_{N}$ as the fundamental group of a finite graph of groups with trivial edge groups. They also prove the existence of such "foldable maps" in that setting. The general definition and construction of foldable maps are fairly complicated, but in the context of $\mathcal{A}$ graphs corresponding to markings on $F_{N}$ they become much easier. In particular, we will only need the following basic fact that follows directly from comparing Definition 5.6 with the Handel-Mosher definition of a foldable map:

Let $\Gamma$ be a finite connected $\mathcal{A}$-graph such that the natural projection $p: \Gamma \rightarrow R_{\mathcal{A}}$ is a homotopy equivalence and such that $\Gamma=\operatorname{Core}(\Gamma)$. Suppose that $p$ is foldable in the sense of Definition 5.6 above. Then there exists a foldable (in the sense of Handel-Mosher) map $\widetilde{\Gamma} \rightarrow \widetilde{R}_{\mathscr{A}}$.

Handel and Mosher use foldable maps as a starting point in constructing folding paths between vertices of $\mathrm{FS}_{N}^{\prime}$, and we will need the above fact in the proof of the main result in Section 6 .

\section{Proof of the main result}

Before giving a proof of the main result, we illustrate the relationship and the maps between $\mathrm{FS}_{N}, \mathrm{FS}_{N}^{\prime}, \mathrm{FF}_{N}$ and $\mathrm{FB}_{N}$ in the following diagram, provided by the referee: 


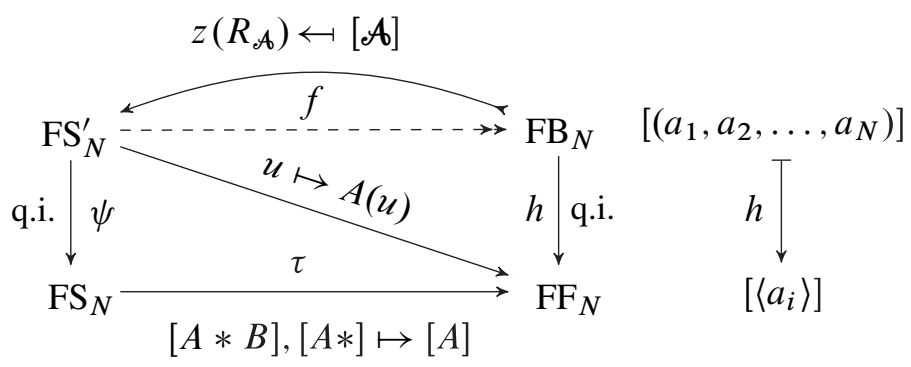

Figure 1. A diagram showing the maps between the various curve complex analogs considered in this paper. Here $F_{N}=A *$ represents an HNN-extension splitting of $F_{N}$ with the trivial associated subgroups and with the base group $A$. The map $\psi$ is the quasi-isometry between $\mathrm{FS}_{N}^{\prime}$ and $\mathrm{FS}_{N}$ given by the inclusion map.

Theorem 6.1. Let $N \geq 3$. Then the free factor complex $\mathrm{FF}_{N}$ is Gromov-hyperbolic. Moreover, there exists a constant $H>0$ such that for any two vertices $x, y$ of $\mathrm{FS}_{N}$ and any geodesic $[x, y]$ in $\mathrm{FS}_{N}^{(1)}$ the path $\tau([x, y])$ is H-Hausdorff close to a geodesic $[\tau(x), \tau(y)]$ in $\mathrm{FF}_{N}^{(1)}$.

Proof. Recall that $\mathrm{FS}_{N}^{\prime}$ is the barycentric subdivision of the free splitting graph $\mathrm{FS}_{N}$, so that the inclusion map $\mathrm{FS}_{N} \subseteq \mathrm{FS}_{N}^{\prime}$ is a quasi-isometry.

Recall also that for any free basis $\mathcal{A}$ of $F_{N}$ the rose $R_{\mathcal{A}}$ defines an $(N-1)$-simplex in $\mathrm{FS}_{N}$ (via the canonical marking $F_{N} \rightarrow \pi_{1}\left(R_{\mathscr{A}}\right)$ sending the elements of $\mathcal{A}$ to the corresponding loop-edges of $\left.R_{\mathscr{A}}\right)$ and that, as in Convention 3.4, $z\left(R_{\mathscr{A}}\right) \in V\left(\mathrm{FS}_{N}^{\prime}\right)$ is the barycenter of that simplex. Note that by definition, if $[\mathcal{A}]=[\mathscr{B}]$ then $z\left(R_{\mathscr{A}}\right)=$ $z\left(R_{\mathscr{B}}\right)$. Put

$$
S=\left\{z\left(R_{\mathcal{A}}\right) \mid \mathcal{A} \text { is a free basis of } F_{N}\right\} .
$$

Thus $S \subseteq V\left(\mathrm{FS}_{N}^{\prime}\right)$ and we may think of $S$ as a copy of $V\left(\mathrm{FB}_{N}\right)$ in $V\left(\mathrm{FS}_{N}^{\prime}\right)$.

For every $x, y \in S$ let $g_{x, y}$ be the path from $x$ to $y$ in $\mathrm{FS}_{N}^{\prime}$ given by the HandelMosher folding line [16]. Recall that, as proved in [16], $\mathrm{FS}_{N}^{\prime}$ is Gromov-hyperbolic and $g_{x, y}$ is a re-parameterized uniform quasigeodesic. Hence $g_{x, y}$ is uniformly Hausdorff close to any geodesic $[x, y]$ in $\mathrm{FS}_{N}^{\prime}$.

Consider the following map $f: V\left(\mathrm{FS}_{N}^{\prime}\right) \rightarrow V\left(\mathrm{FB}_{N}\right)$. For every vertex $u$ of $\mathrm{FS}_{N}^{\prime}$, which may be viewed as a splitting of $F_{N}$ as the fundamental group of a graph of groups with trivial edge-groups, choose an edge $e$ of that splitting, collapse the rest of $u$ to a single-edge splitting corresponding to $u$ and let $A(u)$ be a vertex group of that collapsed splitting. Thus $A(u)$ is a proper free factor of $F_{N}$ and hence $[A(u)]$ is a vertex of $\mathrm{FF}_{N}$. Then choose a vertex $v$ of $\mathrm{FB}_{N}$ with $d(h(v),[A(u)]) \leq 3$ (such $v$ exists by Proposition 4.3). Put $f(u):=v$. We can make the above choices to make sure that for every free basis $\mathcal{A}$ of $F_{N}$ we have $f\left(z\left(R_{\mathscr{A}}\right)\right)=[\mathcal{A}]$ (note that 
$\left.d_{\mathrm{FF}_{N}}\left(h([\mathcal{A}]), A\left(z\left(R_{\mathcal{A}}\right)\right)\right) \leq 2\right)$. With the above mentioned identification of $S$ and $V\left(\mathrm{FB}_{N}\right)$ we may in fact informally think that $\left.f\right|_{S}=\operatorname{Id}_{S}$. Moreover, if $\Gamma$ is a foldable $\mathcal{A}$-graph (which therefore defines a marking $\bar{\Gamma}$ ) and $T \subseteq \Gamma$ is a maximal tree, then we have an associated free basis $\mathcal{B}(\Gamma, T)$ described in Remark 5.5 above. One can check that $d(f(\bar{\Gamma}),[\mathcal{B}(\Gamma, T)]) \leq B$ for some constant $B=B(N)>0$ independent of $\mathcal{A}, \Gamma, T$.

We have defined a map $f: V\left(\mathrm{FS}_{N}^{\prime}\right) \rightarrow V\left(\mathrm{FB}_{N}\right)$. We then extend this map to a graph-map $f: \mathrm{FS}_{N}^{\prime} \rightarrow \mathrm{FB}_{N}$ by sending an arbitrary edge $e$ of $\mathrm{FS}_{N}^{\prime}$ with endpoints $u_{1}, u_{2}$ to a geodesic edge-path $\left[f\left(u_{1}\right), f\left(u_{2}\right)\right]$ in $\mathrm{FB}_{N}$. The graph-map $f: \mathrm{FS}_{N}^{\prime} \rightarrow$ $\mathrm{FB}_{N}$ is $L$-Lipschitz for some $L \geq 0$, since $\tau$ is Lipschitz and the maps $V\left(\mathrm{FS}_{N}^{\prime}\right) \rightarrow$ $\mathrm{FF}_{N}^{(1)}$, given by $u \mapsto[A(u)]$ and $u \mapsto \tau(u)$, are bounded distance away from each other.

We claim that all the assumptions of Proposition 2.5 are satisfied for the map $f: \mathrm{FS}_{N}^{\prime} \rightarrow \mathrm{FB}_{N}$ and the set $S$.

Condition (1) of Proposition 2.5 holds, since by assumption $f(S)=V\left(\mathrm{FB}_{N}\right)$. Also, as noted above, $f: \mathrm{FS}_{N}^{\prime} \rightarrow \mathrm{FB}_{N}$ is $L$-Lipschitz, and it is easy to see that $S$ is $D$-dense in $\mathrm{FS}_{N}^{\prime}$, for some $D>0$. Our task is to verify condition (3) of Proposition 2.5.

If $x^{\prime}, y^{\prime} \in V\left(\mathrm{FB}_{N}\right)$ have $d\left(x^{\prime}, y^{\prime}\right) \leq 1$ then there exist free bases $\mathcal{A}, \mathscr{B}$ of $F_{N}$ such that $x^{\prime}=[\mathscr{B}], y^{\prime}=[\mathcal{A}]$ and such that there exists $a \in \mathcal{A} \cap \mathscr{B}$. Without loss of generality, we may assume that $\mathcal{A}=\left\{a_{1}, \ldots, a_{N}\right\}, \mathscr{B}=\left\{b_{1}, \ldots, b_{N}\right\}$ and that $a_{1}=b_{1}=a$. Put $x=z\left(R_{\mathcal{B}}\right)$ and $y=z(\mathcal{A})$, so that $f(x)=x^{\prime}$ and $f(y)=y^{\prime}$.

In [16] Handel-Mosher [16], given any ordered pair of vertices $x, y$ of $V\left(\mathrm{FS}_{N}^{\prime}\right)$, construct an edge-path $g_{x, y}$ from $x$ to $y$ in $\mathrm{FS}_{N}^{\prime}$, which we will sometimes call the Handel-Mosher folding path. The general definition of $g_{x, y}$ in [16] is fairly complicated. However, we only need to use this definition for the case where $x, y \in S$, in which case it becomes much simpler, and which we will now describe in greater detail for the vertices $x=z\left(R_{\mathscr{B}}\right)$ and $y=z(\mathcal{A})$ defined above.

Consider an $\mathcal{A}$-graph $\Gamma_{0}$ which is a wedge of $N$ simple loops at a common basevertex $v_{0}$, where the $i$-th loop is labeled by the freely reduced word over $\mathcal{A}$ that is equal to $b_{i}$ in $F_{N}$. Note that the first loop is just a loop-edge labelled by $a_{1}$, since by assumption $b_{1}=a_{1}$. The natural projection $p: \Gamma_{0} \rightarrow R_{\mathscr{A}}$ is a homotopy equivalence and we also have $\Gamma_{0}=\operatorname{Core}\left(\Gamma_{0}\right)$. Condition (1) of Definition 5.6 holds for $\Gamma_{0}$ by construction. However, $p: \Gamma_{0} \rightarrow R_{\mathscr{A}}$ is not necessarily foldable since Condition (2) of Definition 5.6 may fail. This happens exactly when there exists $\varepsilon \in\{1,-1\}$ such that for all $i=2, \ldots, N$ the freely reduced word over $\mathcal{A}$ representing $b_{i}$ begins with $a_{1}^{\varepsilon}$ and ends with $a_{1}^{-\varepsilon}$. However, after possibly replacing $\mathscr{B}$ by an equivalent free basis of the form $a_{1}^{m} \mathscr{B} a_{1}^{-m}$, for the graph $\Gamma_{0}$ defined as above the natural projection $p: \Gamma_{0} \rightarrow R_{\mathscr{A}}$ is foldable in the sense of Definition 5.6. Note that conjugation by $a_{1}^{m}$ fixes the element $b_{1}=a_{1}$, so that even after the above modification of $\mathscr{B}$ it will still be true that $\Gamma_{0}$ contains a loop-edge at $v_{0}$ with label $a_{1}$.

As noted in Remark 5.7 above, as the initial input for constructing $g_{x, y}$, Handel 
and Mosher need a "foldable" (in the sense of [16]) $F_{N}$-equivariant map $\widetilde{R_{B}} \rightarrow \widetilde{R_{\mathcal{A}}}$. Again, as observed in Remark 5.7, such a map exists since we have arranged for the $\mathcal{A}$-graph $\Gamma_{0}$ to be foldable in the sense of Definition 5.6.

Note that by construction, the marking $\overline{\Gamma_{0}}$ corresponding to $\Gamma_{0}$ is exactly the vertex $x=z\left(R_{B}\right)$ of $\mathrm{FS}_{N}^{\prime}$.

The remainder of the Handel-Mosher construction of $g_{x, y}$ in this case works as follows. Since $p: \Gamma_{0} \rightarrow R_{\mathcal{A}}$ is a homotopy equivalence, there exists a finite sequence of $\mathcal{A}$-graphs

$$
\Gamma_{0}, \Gamma_{1}, \ldots \Gamma_{n}=R_{\mathcal{A}}
$$

where for $i=1, \ldots, n \Gamma_{i}$ is obtained from $\Gamma_{i-1}$ by a maximal fold. Then the associated markings $\bar{\Gamma}_{i}$ are vertices of $\mathrm{FS}_{N}^{\prime}$. As observed in Remark 5.7, we have $d\left(\overline{\Gamma_{i-1}}, \overline{\Gamma_{i}}\right) \leq 2$ in $\mathrm{FS}_{N}^{\prime}$. Joining each consecutive pair $\overline{\Gamma_{i-1}}, \overline{\Gamma_{i}}$ by a geodesic path of length $\leq 2$ in $\mathrm{FS}_{N}^{\prime}$ produces the path $g_{x, y}$ from $x$ to $y$ in $\mathrm{FS}_{N}^{\prime}$. Note that each $\Gamma_{i}$ has a base-vertex $v_{i}$ which is the image of the base-vertex $v_{0}$ of $\Gamma_{0}$ under the sequence of folds that takes $\Gamma_{0}$ to $\Gamma_{i}$.

A crucial feature of the above construction is that every $\Gamma_{i}$ will have a loop-edge (at the base-vertex $v_{i}$ of $\Gamma_{i}$ ) with label $a_{1}$. Since the map $f: \mathrm{FS}_{N}^{\prime} \rightarrow \mathrm{FB}_{N}$ is $L$-Lipschitz, this implies that $f\left(g_{x, y}\right)$ has diameter bounded by some constant $M_{0}$ independent of $x, y$. Indeed, Since $\Gamma_{i}$ has a loop-edge at its base-vertex with label $a_{1}$, there exists a free basis $\gamma_{1}, \ldots, \gamma_{N}$ of $\pi_{1}\left(\Gamma_{i}, v_{i}\right)$ (e.g. coming from a choice of a maximal tree in $\Gamma_{i}$, as in Definition 4.4 and Remark 5.5) such that $\mu\left(\gamma_{1}\right)=a_{1}$ and such that $\mathscr{B}_{i}=\left\{\mu\left(\gamma_{1}\right), \ldots, \mu\left(\gamma_{N}\right)\right\}$ is a free basis of $F_{N}$. Since $a_{1} \in \mathscr{B}_{i}$, we have $d\left(\left[\mathscr{B}_{i}\right],[\mathcal{A}]\right) \leq 1$ in $\mathrm{FB}_{N}$ for each $i$. Unpacking the definition of the map $f$ we see that $d\left(f\left(\overline{\Gamma_{i-1}}\right),\left[\mathscr{B}_{i}\right]\right) \leq C$ in $\mathrm{FB}_{N}$ for some constant $C \geq 0$. Hence $d\left(f\left(\overline{\Gamma_{i-1}}\right),[\mathcal{A}]\right) \leq C+1$. Recall that $g_{x, y}$ is a quasi-geodesic in a hyperbolic graph $\mathrm{FS}_{N}^{\prime}$ and hence $g_{x, y}$ is uniformly Hausdorff-close to a geodesic $[x, y]$. Since $f$ is $L$-Lipschitz, it follows that $f([x, y])$ has diameter bounded by some constant $M$ independent of $x, y$. Thus condition (3) of Proposition 2.5 holds.

Therefore, by Proposition 2.5, the graph $\mathrm{FB}_{N}$ is Gromov-hyperbolic, and, moreover, for any vertices $x, y$ of $\mathrm{FS}_{N}$, the path $f([x, y])$ is uniformly Hausdorff-close to a geodesic $[f(x), f(y)]$.

Recall that in Proposition 4.3 we constructed an explicit quasi-isometry $h: \mathrm{FB}_{N} \rightarrow$ $\mathrm{FF}_{N}$. Since $\mathrm{FB}_{N}$ is hyperbolic, it follows that $\mathrm{FF}_{N}$ is Gromov-hyperbolic as well. Moreover, the map $\tau: \mathrm{FS}_{N} \rightarrow \mathrm{FF}_{N}$ from the statement of Theorem 1.1, and the map $h \circ f: \mathrm{FS}_{N} \rightarrow \mathrm{FF}_{N}$ are bounded distance from each other. This implies that there exists a constant $H>0$ such that for any two vertices $x, y$ of $\mathrm{FS}_{N}$ and any geodesic $[x, y]$ in $\operatorname{FS}_{N}^{(1)}$ the path $\tau([x, y])$ is $H$-Hausdorff close to a geodesic $[\tau(x), \tau(y)]$ in $\mathrm{FF}_{N}^{(1)}$.

Remark 6.2. The above proof implies a reasonably explicit description of certain reparameterized quasigeodesics in $\mathrm{FB}_{N}$ between two arbitrary vertices of $\mathrm{FB}_{N}$ in terms of Stallings folds. Let $\mathcal{A}=\left\{a_{1}, \ldots, a_{N}\right\}$ and $\mathcal{B}=\left\{b_{1}, \ldots, b_{N}\right\}$ be free bases 
of $F_{N}$. Let $\Gamma_{0}$ be an $\mathcal{A}$-graph corresponding to $\mathscr{B}$ constructed in a similar way to the way $\Gamma_{0}$ was constructed in the above proof. That is, let $\Gamma_{0}$ be a wedge of $N$ simple loops at a common base-vertex $v_{0}$, where the $i$-th loop is labeled by the freely reduced word over $\mathcal{A}$ that is equal to $b_{i}$ in $F_{N}$. Suppose that $\Gamma_{0}$ is such that the natural projection $p: \Gamma_{0} \rightarrow R_{\mathcal{A}}$ is foldable in the sense of Definition 5.6. (Note that this assumption does not always hold; however, it may always be ensured after replacing $\mathscr{B}$ by an equivalent free basis).

Let $\Gamma_{0}, \Gamma_{1}, \ldots, \Gamma_{n}=R_{\mathcal{A}}$ be $\mathcal{A}$-graphs such that for $i=1, \ldots, n \Gamma_{i}$ is obtained from $\Gamma_{i-1}$ by a maximal fold. Note that each $\Gamma_{i}$ has a distinguished base-vertex $v_{i}$, which is the image of the base-vertex $v_{0}$ of $\Gamma_{0}$ under the foldings transforming $\Gamma_{0}$ to $\Gamma_{i}$.

For each $1 \leq i<n$ choose a maximal subtree $T_{i}$ in $\Gamma_{i}$. Let $\mathcal{A}_{i}=\mathscr{B}\left(\Gamma_{i}, T_{i}\right)$ be the associated free basis of $F_{N}$ (see Remark 5.5 above for its detailed description). Put $\mathcal{A}_{0}=\mathscr{B}$ and $\mathcal{A}_{n}=\mathcal{A}_{\text {. }}$

It is not hard to check that $d\left(\left[\mathcal{A}_{i}\right], f\left(\bar{\Gamma}_{i}\right)\right) \leq C$ in $\mathrm{FB}_{N}$ for some constant $C=$ $C(N)>0$ independent of $\mathcal{A}, \mathcal{B}$. Since, as noted in the proof of Theorem 1.1 above, the sequence $\overline{\Gamma_{0}}, \ldots, \overline{\Gamma_{n}}$ defines a (reparameterized) uniform quasigeodesic in $\mathrm{FS}_{N}^{\prime}$, it now follows from the proof of Theorem 1.1 that the set $\left\{\left[\mathcal{A}_{0}\right],\left[\mathcal{A}_{1}\right], \ldots,\left[\mathcal{A}_{n}\right]\right\}$ is uniformly Hausdorff-close to a geodesic joining $[\mathscr{B}]$ and $[\mathcal{A}]$ in $\mathrm{FB}_{N}$. This fact can also be derived from a careful analysis of the Bestvina-Feighn proof [4] of hyperbolicity of $\mathrm{FF}_{N}$.

\section{References}

[1] J. Aramayona and J. Souto, Automorphisms of the graph of free splittings. Michigan Math. J. 60 (2011), no. 3, 483-493. Zbl 1242.05117 MR 2861084

[2] J. Behrstock, M. Bestvina, and M. Clay, Growth of intersection numbers for free group automorphisms. J. Topol. 3 (2010), no. 2, 280-310. Zbl 1209.20031 MR 2651361

[3] M. Bestvina, and M. Feighn, A hyperbolic Out $\left(F_{n}\right)$-complex. Groups Geom. Dyn. 4 (2010), no. 1, 31-58. Zbl 1190.20017 MR 2566300

[4] M. Bestvina, and M. Feighn, Hyperbolicity of the complex of free factors. Adv. Math. 256 (2014), 104-155; corrigendum, ibid. 259 (2014), 843. Zbl 06284922 MR 3177291

[5] M. Bestvina, and M. Feighn, Subfactor projections. Preprint, arXiv:1211.1730.

[6] M. Bestvina and P. Reynolds, The boundary of the complex of free factors. Preprint, arXiv:1211.3608.

[7] B. Bowditch, Relatively hyperbolic groups. Internat. J. Algebra Comput. 22 (2012), no. 3, 1250016, 66 pp. Zbl 1259.20052 MR 2922380

[8] B. Bowditch, Intersection numbers and the hyperbolicity of the curve complex. J. Reine Angew. Math. 598 (2006), 105-129. Zbl 1119.32006 MR 2270568

[9] B. Bowditch, Uniform hyperbolicity of the curve graphs. Pacific J. Math., to appear. 
[10] J. Brock, R. Canary, and Y. Minsky, The classification of Kleinian surface groups, II: The ending lamination conjecture. Ann. of Math. (2) 176 (2012), no. 1, 1-149. Zbl 1253.57009 MR 2925381

[11] M. Culler and K. Vogtmann, Moduli of graphs and automorphisms of free groups. Invent. Math. 84 (1986), no. 1, 91-119 Zbl 0589.20022 MR 0830040

[12] F. Dahmani and V. Guirardel, Rotating families, and the structure of some normal subgroups in groups acting on hyperbolic spaces. Preprint, http://perso.univ-rennes1.fr/vincent.guirardel/papiers/rotating.pdf.

[13] B. Farb, Relatively hyperbolic groups. Geom. Funct. Anal. 8 (1998), no. 5, 810-840. Zbl 0985.20027 MR 1650094

[14] U. Hamenstädt, Geometry of the complex of curves and of Teichmüller space. In Handbook of Teichmüller Theory, Vol. 1, A. Papadopoulos, ed., European Math. Soc., Zürich 2007, 447-467. Zbl 1162.32010 MR 2349677

[15] U. Hamenstädt, The boundary of the free splitting graph and the free factor graph. Preprint, arXiv:1211.1630.

[16] M. Handel and L. Mosher, The free splitting complex of a free group I: Hyperbolicity. Geom. Topol. 17 (2013), no. 3, 1581-1672. Zbl 1278.20053 MR 3073931

[17] W. J. Harvey, Boundary structure of the modular group. In Riemann surfaces and related topics: Proceedings of the 1978 Stony Brook Conference (State Univ. New York, Stony Brook, N.Y., 1978), Ann. of Math. Stud. 97, Princeton University Press, Princeton, N.J., 1981, 245-251. Zbl 0461.30036 MR 0624817

[18] A. Hatcher and K. Vogtmann, The complex of free factors of a free group. Quart. J. Math. Oxford Ser. (2) 49 (1998), no. 196, 459-468. Zbl 0935.20015 MR 1660045

[19] A. Hilion and C. Horbez, The hyperbolicity of the sphere complex via surgery paths. Preprint, arXiv:1210.6183.

[20] I. Kapovich and M. Lustig, Geometric intersection number and analogues of the curve complex for free groups. Geom. Topol. 13 (2009), no. 3, 1805-1833. Zbl 1194.20046 MR 2496058

[21] I. Kapovich and A. Myasnikov, Stallings foldings and subgroups of free groups. J. Algebra 248 (2002), no. 2, 608-668. Zbl 1001.20015 MR 1882114

[22] J. Maher and S. Schleimer, The graph of handlebodies. In preparation.

[23] H. Masur, and Y. Minsky, Geometry of the complex of curves. I. Hyperbolicity. Invent. Math. 138 (1999), no. 1, 103-149. Zbl 0941.32012 MR 1714338

[24] H. Masur, and Y. Minsky, Geometry of the complex of curves. II. Hierarchical structure. Geom. Funct. Anal. 10 (2000), no. 4, 902-974. Zbl 0972.32011 MR 1791145

[25] H. Masur and S. Schleimer, The geometry of the disk complex. J. Amer. Math. Soc. 26 (2013), no. 1, 1-62. Zbl 1272.57015 MR 2983005

[26] Y. Minsky, End invariants and the classification of hyperbolic 3-manifolds. In Current developments in mathematics, 2002, International Press, Somerville, Mass., 2003, 181217. Zbl 1049.57010 MR 2062319

[27] Y. Minsky, The classification of Kleinian surface groups. I. Models and bounds. Ann. of Math. (2) 171 (2010), no. 1, 1-107. Zbl 1193.30063 MR 2630036 
[28] M. Mj and L. Reeves, A combination theorem for strong relative hyperbolicity. Geom. Topol. 12 (2008), no. 3, 1777-1798. Zbl 1192.20027 MR 2421140

[29] L. Sabalka, and D. Savchuk, On the geometry of a proposed curve complex analogue for $\operatorname{Out}\left(F_{n}\right)$. Preprint, arXiv:1007.1998.

[30] L. Sabalka, and D. Savchuk, Submanifold projection. Preprint, arXiv:1211.3111.

[31] J. R. Stallings, Topology of finite graphs. Invent. Math. 71 (1983), no. 3, 551-565 Zbl 0521.20013 MR 0695906

Received September 11, 2012; revised November 30, 2012

I. Kapovich, Department of Mathematics, University of Illinois at Urbana-Champaign, 1409 West Green Street, Urbana, IL 61801, U.S.A.

E-mail: kapovich@math.uiuc.edu

K. Rafi, Department of Mathematics, University of Oklahoma, Norman, OK 73019, U.S.A.

E-mail: rafi@math.ou.edu 